\title{
Prediction of tool tip dynamics for generalized milling cutters using the $3 D$ model of the tool body
}

\section{Lutfi Taner Tunc}

The International Journal of Advanced Manufacturing Technology ISSN 0268-3768

Int J Adv Manuf Technol DOI 10.1007/s00170-017-1286-X

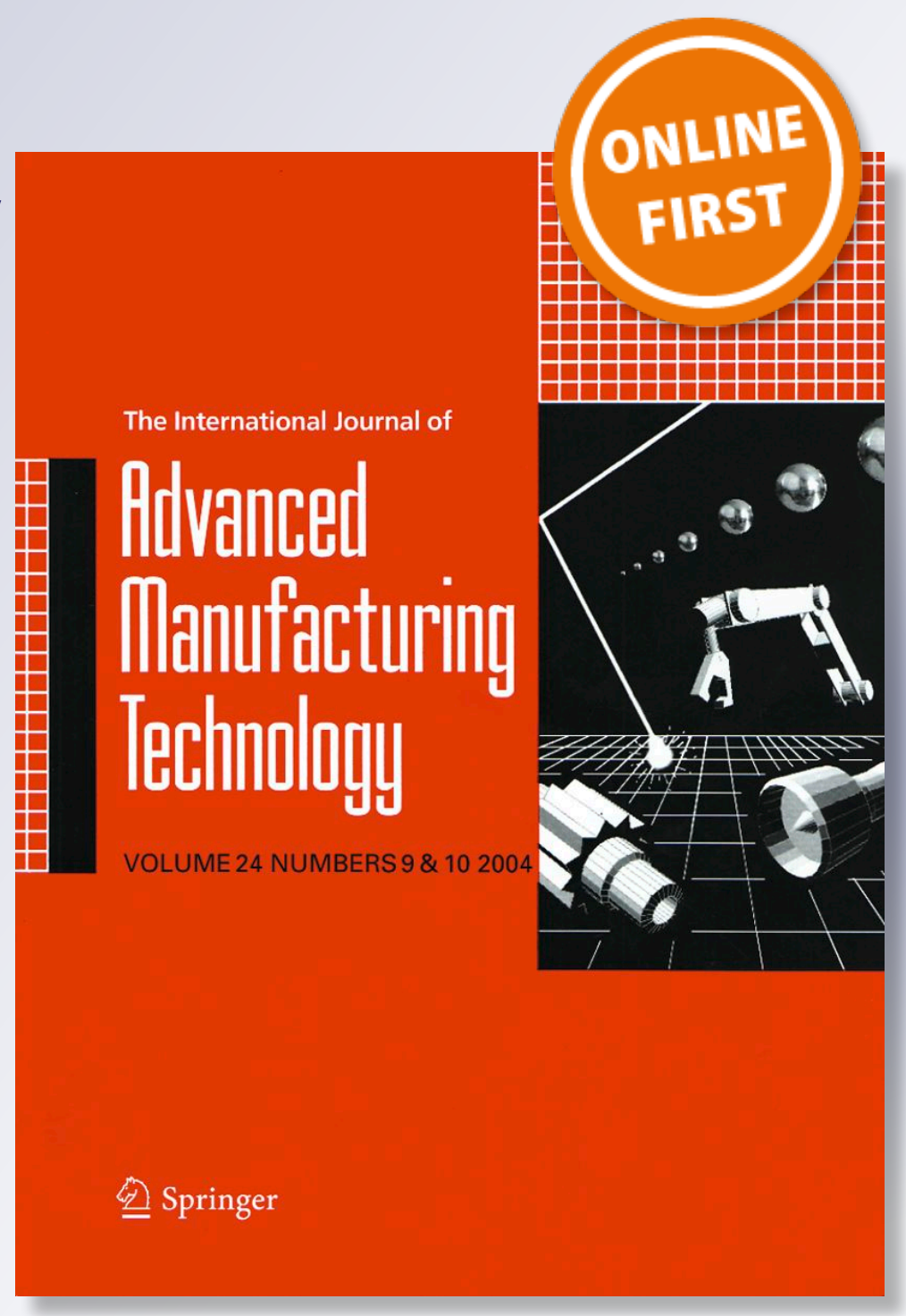

黛 Springer 
Your article is protected by copyright and all rights are held exclusively by SpringerVerlag London Ltd.. This e-offprint is for personal use only and shall not be selfarchived in electronic repositories. If you wish to self-archive your article, please use the accepted manuscript version for posting on your own website. You may further deposit the accepted manuscript version in any repository, provided it is only made publicly available 12 months after official publication or later and provided acknowledgement is given to the original source of publication and a link is inserted to the published article on Springer's website. The link must be accompanied by the following text: "The final publication is available at link.springer.com". 


\title{
Prediction of tool tip dynamics for generalized milling cutters using the 3D model of the tool body
}

\author{
Lutfi Taner Tunc ${ }^{1,2}$
}

Received: 30 May 2017 / Accepted: 27 October 2017

(C) Springer-Verlag London Ltd. 2017

\begin{abstract}
In general, chatter is the main limitation to proper material removal in milling operations. Stability lobes are good tools to determine chatter-free cutting conditions in terms of spindle speed and cutting depth, which require the frequency response function (FRF) at the tool tip to be known. There are experimental methods to measure the tool tip FRF but this may be time consuming or even impossible for each tool and tool holder combination. Receptance coupling substructure analysis (RCSA) is a widely used approach to predict tool tip dynamics. This paper proposes the use of the RCSA approach with a stereolithographic (STL) slicing algorithm to enable the exact calculation of cross sectional properties such as area and area moment of inertia of the cutting tool from its 3D model opposed to the approximation methods. So that, the effect of flutes on cutting tool structure introduced in an exact manner and the RCSA approach becomes feasible for more complicated tool geometries with varying cross-sectional properties, i.e., tapered ball end mills, end mills with variable flute geometries, and so on. The solid model of the tool can be available by either the tool manufacturer or 3D measurement. Although, at the presence of 3D models, finite element methods (FEM) offer accurate simulation of the dynamic response for solid bodies, they suffer from the compromise between accuracy and computation time, as high number of elements is needed for accuracy. Thus, the use of analytical methods where possible improves the simulation time significantly. The proposed STL slicing
\end{abstract}

Lutfi Taner Tunc

ttunc@sabanciuniv.edu

1 Nuclear Advanced Manufacturing Research Centre, University of Sheffield, Sheffield, UK

2 Composite Technologies Centre of Excellence, Sabanci University, Istanbul, Turkey algorithm is integrated with a previously developed RCSA method. The experimental results show that the proposed algorithm works more accurate in calculation of the cross-sectional properties and hence free-free response of the tool compared to the existing arc approximation methods. It is also shown that the proposed approach performs better than FEM solutions in terms of the computation time and the compromise between accuracy and computation performance. Finally, the proposed approach in prediction of tool tip dynamics for a robotic machining platform.

Keywords Receptance coupling $\cdot$ Milling dynamics $\cdot 3 \mathrm{D}$ tool model $\cdot$ Frequency response function $\cdot$ Stereolithographic (STL)

\section{Introduction}

High speed machining of steel and aluminum alloys has been of high interest in several industries such as nuclear manufacturing, aerospace, automotive, and die-mold. In high speed machining, like most of the metal cutting applications, proper material removal is mostly limited by self-excited vibrations, i.e., chatter, as it causes poor surface finish, mechanical damage to cutting tool, and machine tool components. Stability diagrams are very useful to identify the chatter-free cutting conditions.

Altintas and Budak [1] proposed the very first zeroth order approximation of the time periodic milling system to solve the stability problem for regular end milling operations. Then, Insperger and Stepan [2] proposed the semi discretization approach to solve the stability problem for milling operations where milling tools with irregular edge geometries such as variable helix, and variable pitch introduce multiple time delays to the process. They demonstrated successful application of the semi discretization method through accurate 
experimental verification. Prediction of stable cutting conditions, either in frequency domain or in time domain requires the dynamic response at the tool tip to be known in the form of frequency response function (FRF) or modal parameters. Experimental methods such as impact hammer tests are widely used in identification of tool tip dynamics, which might be time consuming or even impossible considering the infinitely many number of tool and tool holder combinations. To minimize the costly experiments, prediction of tool tip dynamics has caught a strong interest in the literature.

Towards prediction of tool tip FRF, Schmitz and Donaldson [3] successfully introduced and applied the receptance coupling substructure analysis (RCSA). They coupled the measured spindle tip FRF with the free-free response of the tool by numerically calibrating the connection parameters at the tool-tool holder interface. In the literature, Euler-Bernoulli and Timoshenko beam theories are used to analytically predict the free-free response of the milling tool body. Aristizabal-Ochoa [4] proposed a solution to Timoshenko beam with generalized end conditions, which is later found to be suitable to simulate dynamic response of milling tools. Schmitz and Donaldson [3] proposed the first RCSA model to predict the tool tip FRF, where the free-free tool body is modeled as an EulerBernoulli beam. However, they ignored the rotational degrees of freedom (DOF) at the tool-tool holder interface. Then, Schmitz et al. [5] used RCSA to improve the high-speed material removal rates, by optimizing the tool length for higher stability limits. This is later improved by including the rotational DOF related to bending.

After realizing the effectiveness of RCSA, several authors further studied to improve the accuracy of RCSA with a focus to identify the tool-tool holder connection parameters and introduction of rotational DOF. One of the first methods to identify joint parameters is proposed by Park et al. [6] based on inverse receptance coupling. In their study, a rigid joint representing the flexibility of the connection is considered by the impact hammer tests done on the blanks having different lengths. This new method to identify the dynamics on the tool holder tip also showed the effect of rotational DOF on the accuracy of simulations. The effect of cutting flutes on the free-free tool FRF is introduced by considering an equivalent radius at the fluted section, which is $80 \%$ of the tool radius, in the developed FEM model. However, this geometrical approximation may lead to inaccurate results. Considering that crosssectional area and moment of inertia are function of 2 nd and 4th power of diameter, respectively selection of equivalent diameter might be an issue. Schmitz et al. [7] worked on identification of stiffness and damping parameters for shrink fit tool holders. Then, Namazi et al. [8] proposed a technique to identify the tool holder-spindle interface dynamics towards extension of the RCSA method. They modeled the holder-spindle interface as uniformly distributed linear and rotational springs. As outcome of this study, the RCSA method was extended to prediction of tool tip FRF by coupling the spindle, tool holder and cutting tool dynamics. This technique was further improved by Yang et al. [9] to better predict the dynamics introduced by the collet geometry to the tool tip FRF. They used Euler-Bernoulli beam elements to model the tool-collet and holder-collet joint interfaces. With a similar focus, Ozsahin et al. [10], proposed a closed form solution approach to identify the dynamic contact parameters in spindle-holder-tool assemblies. This was a good step ahead towards prediction of tool tip dynamics when the spindle-holder-tool geometries and contact parameters were known. In a two-part study Erturk et al. [11, 12] proposed one of the first complete analytical model to predict spindletool dynamics based on Timoshenko beam theory, through RCSA. This study extended the use of Timoshenko beam and RCSA to modeling of spindle dynamics. Albertelli et al. [13] proposed a new RCSA methodology to overcome the difficulties in the estimation of rotational dynamics. They proposed an FEM based approach, where the free-free response of the tool is used a reference to calibrate the dynamics due to the rotational degrees of freedom.

Kivanc and Budak [14] proposed the first analytical model to include the geometrical effect of cutting flutes on tool structure, where they utilized Euler-Bernoulli beam to predict static and dynamic response. The gash geometry at the cutting flutes is analytically approximated in the form of a circular arc with equivalent radius. The area and moment of inertia is analytically modeled for 4-flute, 3-flute, and 2-flute end mills. Although, they demonstrated the use of the method by successful experimental verification, this approach requires several geometrical parameters to be identified to model the approximated circular arc representing the cutting flutes. This approach is practical for approximation of the cross-sectional properties of the fluted part of regular end mills but it becomes impractical for tools having variable cross section, i.e., tapered mills. The analytical approximation of flute geometry is further used by Mancisidor et al. [15], where a new Timoshenko beam model was proposed by use of the fixed boundaries approach as opposed to the use of free-free response of the cutting tool body. Then, Ozsahin and Altintas [16] studied the tool tip FRF prediction for tools having asymmetrical geometry such as 2-fluted ones. They showed the effect of cutting flute geometry on the FRF for asymmetrical tools in two orthogonal directions, i.e., $\mathrm{x}$ and $\mathrm{y}$. They pointed out the importance of accurate modeling and treatment of tool geometry in prediction FRF in two orthogonal directions, especially for such type of milling tools. In one of the recent studies, Yamg et al. [17] presented a generalized method to model the FRF at the tip of rotation tools such as drills, milling tools, and boring bars. They included the translational and rotational DOF along each direction, i.e., X, Y, and $\mathrm{Z}$. As a result, the bending, torsional, and axial responses are obtained. In their model, they considered the tool-holder joint interface as zero-thickness distributed layer, where springdamper elements are used. 
Kivanc and Budak [14] emphasized that modeling the geometrical effect of cutting flutes as one of the challenging issues, besides identification of the connection parameters and structural modeling of the tool's individual response. This becomes even more challenging for tools with varying cross-sectional properties along tool axis, such as tapered milling tools, tools having variable helix, and variable pitch type of cutting edges. In a recent CIRP Keynote paper, Altintas et al. [18] highlighted the significant trend towards virtual manufacturing, where the importance of generalization of the analytical models and utilization of them in a compatible fashion with current CADCAM and NC simulation software systems. In this respect, it can be said that utilizing the 3D CAD models of the cutting tools in process modeling will increase the accuracy and wide spread of the simulations and will bridge the gap from the theoretical models to the virtual manufacturing systems. Moreover, increased ability to use the already available 3D models of the cutting tools may enable tool manufacturers to consider the dynamic response of the tool as a criterion at the tool design phase. In a recent study, Albertelli et al. [13] used the 3D model of the cutting tool in FEM analysis to obtain the dynamics response of the tool body, where the 3D CAD model is discretized into high number of elements. However, FEM may require complex modeling and meshing operations, which makes it difficult to obtain the dynamic response of the tool in a reasonable computation time for quick simulation purposes. In the CAD literature, Lefebvre et al. [19] used STL models for tool path generation in additive manufacturing, where the part geometry was sliced into number of cross sections and the contour was used as the region to be filled with material. This shows that STL slicing is a good way to extract cross-sectional properties of any solid geometry. However, the use of STL models in structural modeling of cutting tools has not been adequately addressed, yet.

The existing literature shows that utilization of accurate analytical models together with precise and generalized geometrical definition of the cutting tool is of great importance in prediction of tool tip FRF. In this paper, a novel STL slicing algorithm is proposed, based on the 3D model of the milling tool, to predict free-free tool FRF not for only flat end mills but for also generalized tool geometries. Overall, the proposed approach is as follows: initially, the tool geometry is obtained in the form of STL triangulation of the solid model including the cutting flutes. Then, the cross-sectional properties, i.e., area and moment of inertia, are evaluated by slicing the STL model at number of axial levels. Each sub segment is modeled as a continuous Timoshenko Beam, which are then coupled. Then, RCSA is used to couple the free-free tool FRF with the spindle dynamics, which is measured at the holder tip in two orthogonal directions. As the first time in the literature, the experimental results of this study show that the use of STL model through the proposed STL slicing algorithm works more accurate, in calculation of the cross-sectional properties and hence the dynamics of the tool body, compared to the existing arc approximation methods. As its performance is compared to the FEM methods in prediction of the tool's free-free dynamic response, it is shown that the calculation speed increases significantly while keeping the accuracy at the same order. The proposed approach relies on analytical beam equations. Thus, once the crosssectional properties are known, it can be used in recursive calculations to optimize several length parameters such as flute length, shank length, and their ratios. For such a purpose, the model eliminates the need for model preparation, model import, and re-meshing phases as in FEM approaches. This advantage turns up to be one of the major benefits over the FEM methods.

Henceforth, the paper is organized as follows: the next section presents the RCSA approach based on the model proposed by Schmitz and Donaldson [3]. The geometric modeling of the cutting tool is given in Section 3, where representative comparisons are provided with the analytical arc approximation. In Section 4, analytical prediction of tool tip FRF is presented based on the Timoshenko beam model as named after the study of Timoshenko [20]. The paper is finalized with experimental verification and conclusions.

\section{Receptance coupling}

The dynamic response of individual components such as spindle, tool holder and tool can be coupled through RCSA to achieve the dynamic response at the tool tip assembly, where the individual dynamic response can be obtained either analytically or experimentally. In this paper, the analytically calculated dynamic response of the cutting tool is coupled with the experimentally obtained dynamic response measured at the tool holder. The cutting tool is divided into sub segments to simulate the effect of varying cross-sectional properties and cutting flutes, which are rigidly coupled to obtain the dynamic response of the cutting tool overhanging outside the tool holder. Then, both responses due to the tool and tool holder are coupled by introducing a rigid joint through the approach proposed by Schmitz and Donaldson [3] as illustrated in Fig. 1.

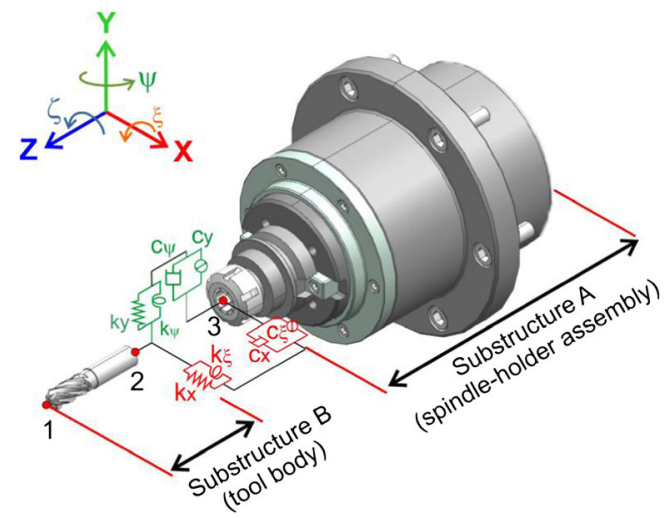

Fig. 1 Coupling of tool holder and tool through RCSA 
The stability models require the transfer function, $\mathrm{G}_{11}$, which is the dynamic response of the tool tip when it is excited. This can be written in any direction in terms of substructure responses according to RCSA.

$$
\left[G_{11}\right]=H_{11}-H_{12} E_{1}^{-1} E_{2}-L_{12} E_{3}^{-1}\left\{\left(k_{\theta} N_{21}+c_{\theta} N_{21}^{\prime}\right)-E_{4} E_{1}^{-1} E_{2}\right\}
$$

where,

$E_{4}=k_{\theta}\left(N_{22}+N_{33}\right)+c_{\theta}\left(N_{22}^{\prime}+N^{\prime}{ }_{33}\right)$

$E_{3}=k_{\theta}\left(P_{22}+P_{33}\right)+c_{\theta}\left(P_{22}^{\prime}+P_{33}^{\prime}\right)+[1]$

$E_{2}=\left(k_{x} H_{21}+c_{x} H_{21}^{\prime}\right)-E_{3}^{-1}\left(k_{\theta} N_{21}+c_{\theta} N^{\prime}{ }_{21}\right)\left\{k_{x}\left(L_{33}+L_{22}\right)+c_{x}\left(L_{33}^{\prime}+L_{22}^{\prime}\right)\right\}$

$E_{1}=\left\{k_{x}\left(H_{22}+H_{33}\right)+c_{x}\left(H_{22}^{\prime}+H_{33}^{\prime}\right)+[1]\right\}-E_{3}^{-1} E_{4}\left(k_{x}\left(L_{22}+L_{33}\right)+c_{x}\left(L_{22}^{\prime}+L_{33}^{\prime}\right)\right)$

In Eq. (1), $H_{i j}, L_{i j}, N_{i j}$, and $P_{i j}$ are the transfer functions measured at point $i$ when the structure is excited at point $j$, relating the applied force to linear displacement, applied moment to linear displacement, applied force to rotational displacement, and applied moment to rotational displacement, respectively. $H_{i j}^{\prime}, L_{i j}^{\prime}, N_{i j}^{\prime}$, and $P_{i j}^{\prime}$ are the mobility transfer functions, which are simply multiplied by $i \omega(\mathrm{rad} / s)$. The dynamics at the tool-tool holder interface is represented by the linear and rotational stiffness and damping terms of $k_{x}, k_{\theta}, c_{x}$, and $c_{\theta}$, which need to be experimentally identified, which is performed through FRF measurements made on tool blanks as detailed in Section 0.

$H_{11}, L_{11}, N_{11}, P_{11}, H_{12}, L_{12}, N_{12}, P_{12}, H_{21}, L_{21}, N_{21}, P_{21}$, $H_{22}, L_{22}, N_{22}$, and $P_{22}$ are the free-free responses of the cutting tool, which are obtained analytically using Timoshenko beam theory as proposed by Timoshenko [20], and Blevins [21]. $H_{33}, L_{33}, N_{33}$, and $P_{33}$ represent the transfer functions to be experimentally identified at the tool holder tip. In this study, the rotational FRFs, i.e., $L_{33}, N_{33}$, and $P_{33}$ are accepted to be zero considering that their effect on the tool tip FRF is not significant as emphasized by Schmitz and Donaldson [3].

\section{Geometric modeling of the cutting tool}

RCSA requires analytical prediction of free-free response of the cutting tool, where the cross-sectional properties such as area and moment of inertia should be known. In general, the tool geometry can be determined by knowing the tool envelope and cutting flute geometry. The tool envelope is the 3D solid geometry generated by the rotation of the tool, where the cutting flute geometry is defined by the number of flutes and the flute edge geometry. In practice, the number of cutting flutes may vary from 2 to 12 and the flute edge geometry may be either regular or irregular. The irregular edge

Fig. 2 3D tool model information

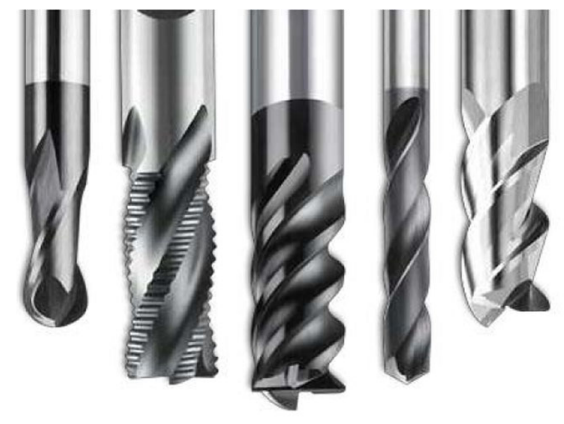

(a) Types of solid milling tools

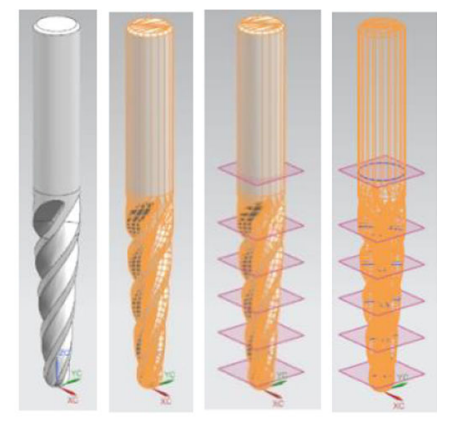

(b) STL slicing

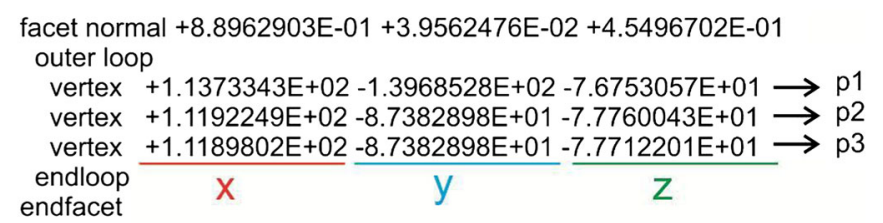

(c) STL file format 
geometries may include variable pitch, variable helix or serrations. The cross-sectional properties vary with the cutting flute geometry, where there may be several tool geometry combinations in milling applications as plenty of them shown in Fig. 2a. Therefore, use of 3D model of the cutting tools, which is available at the discretion of tool manufacturers, is a potential way to generalize the RCSA based analytical modeling approaches for prediction of tool tip response. In this section, geometric modeling of the cutting tool based on the stereolithographic (STL) model slicing as illustrated in Fig. 2b.

\subsection{Working with the STL model}

In this paper, the required cross-sectional information is extracted from the 3D model of the cutting tool including the cutting flutes. The 3D model information is obtained in STL format, where the closed boundary of the 3D tool geometry is stored as number of connected triangles and corresponding unit normal vectors as shown in Fig. 2c. Thus, the information stored in the STL file cannot be directly used in RCSA, which needs to be converted into cross sectional areas along the tool axis. This is done by slicing the STL model into number of planes (see Fig. 2b), and then finding the intersection contour between the axial planes and the triangles representing the boundary of the tool envelope. In this section, the geometrical derivation of the proposed STL slicing algorithm is presented on the complex tool geometry shown in Fig. $2 b$. Thus, the

Fig. 3 STL slicing algorithm. http://en.wikipedia.org/wiki/ Point_in_polygon

illustrations and calculations performed in the rest of this section belong to that specific tool geometry.

In the proposed STL slicing algorithm (see Fig. 3), the number and levels of the slicing planes are decided according to the complexity of the cutting tool. The facets representing the tool boundary are filtered according to $\mathrm{Z}$ levels for each plane (see Fig. 3a). By doing so, the facets matching with the corresponding axial level are determined. At the final step, the intersection points between the filtered facets and the corresponding axial plane need to be calculated to obtain the contour of the cross section. This is performed through planeplane intersection analysis between the axial plane and the edges representing the facet (see Fig. 3b). It is noteworthy to state that the axial plane is an unbounded plane, whereas the facet is bounded by the vertices given in the STL file. Thus, the intersection between the facet and the axial plane must lay on any two of the three edges bounding the facet as illustrated in Fig. 3b. This is checked by calculating the point where the edge pierces the corresponding axial plane using the parametric line definition. In 3D space, a point on a line between two points, from $P_{1}$ to $P_{2}$ is written for parameter $t$ (see Fig. 3b).

$$
\begin{aligned}
& \left\{\begin{array}{l}
x(t) \\
y(t) \\
z(t)
\end{array}\right\}=\left\{\begin{array}{l}
x_{1} \\
y_{1} \\
z_{1}
\end{array}\right\}+t\left\{\begin{array}{l}
x_{2}-x_{1} \\
y_{2}-y_{1} \\
z_{2}-z_{1}
\end{array}\right\} \\
& \text { where, } P_{i}=\left\{\begin{array}{l}
x_{i} \\
y_{i} \\
z_{i}
\end{array}\right\}, i=1,2
\end{aligned}
$$

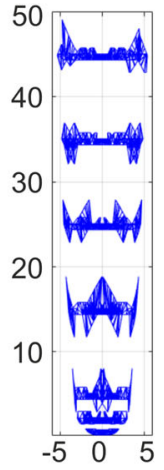

(a) Filtered facets

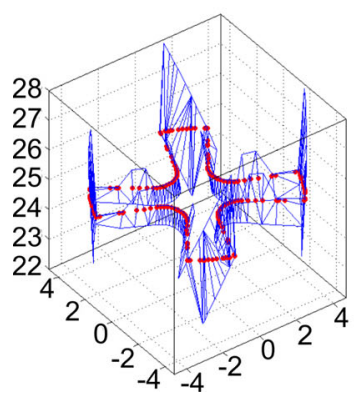

(c) Intersection points

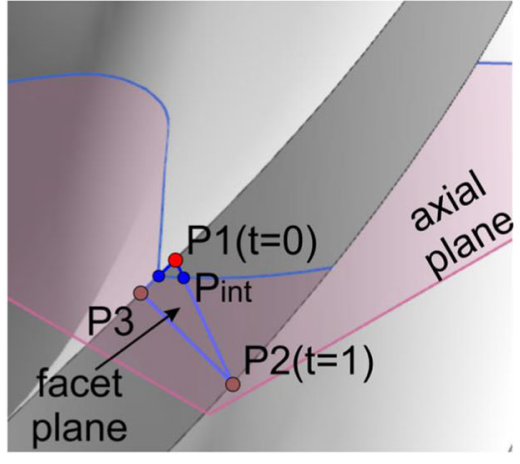

(b) Facet-axial plane intersection

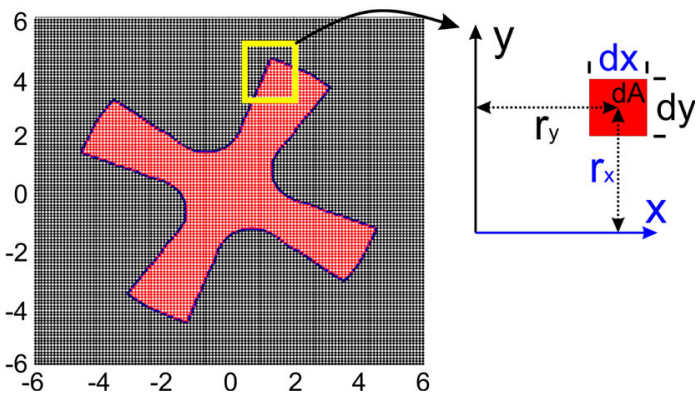

(d) Extracted contour and PIP checking [22]. 
Fig. 4 Flat end mill considered in case 1

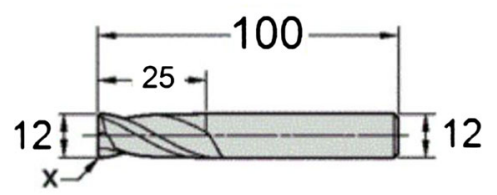

(a) Tool geometry (2CH1200DL022B)

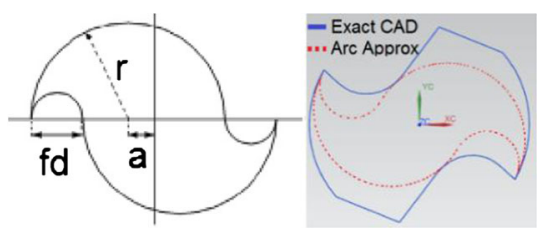

(b) Arc approximation \& Exact-CAD
For an edge bounding, the facet plane to pierce the axial plane, Eq. (2) should be satisfied for values of $t$ in the range of $[0,1]$, which can be determined by inverse solution of Eq. (2). If $t<0$ or $t>1$ the piercing point does not lay between points $P_{1}$ and $P_{2}$. As the $z$ value of the plane, i.e., $z_{\mathrm{p}}$, is known the value of parameter $t_{\text {int }}$, where the edge pierces the plane is calculated as follows:

$t_{\text {int }}=\frac{\left(z_{p}-z_{1}\right)}{\left(z_{2}-z_{1}\right)}$

$t_{\text {int }}$ value is inserted back into Eq. (2) to calculate the point where the edge pierces the corresponding axial plane as illustrated in Fig. 3c. Finally, the piercing points are connected to extract the cross section of the tool body at the level (see Fig. 3d).

\subsection{Calculating the cross-sectional properties}

Once the contours representing the cross section of the tool at each level are determined, it is required to calculate the area bounded by the contour, A, and corresponding area moment of inertia about feed, $x$, and cross feed directions, $y$. This is done by discretizing the bounding box covered by the cross section and identifying the points inside the contour through point-inpolygon (PIP) checking. The contour, points outside the contour (black) and inside the contour (red) are plotted in Fig. 3d for a representative case. After the infinitesimal areas inside the contour are determined, the area of the contour, $\mathrm{A}$, and the moment of inertia, I, are calculated as follows:

$A=\sum_{n=1}^{N} d A=\sum_{n=1}^{N} d x d y$

$I_{i i}=\sum_{n=1}^{N} r_{i, n}^{2} d A$

Fig. 5 Comparison of calculated section properties where, $n$ is the index of the discretized area, $N$ is the number of points, $r_{i, n}$ is the distance of a discretized area to the principal axis $i$.

Having discussed the calculation of cross sectional properties, in the rest of this section the accuracy of the proposed approach is evaluated through a representative case study. Firstly, the calculated cross-sectional properties are compared to the exact values obtained from the CAD software NX8.5 @ Then, a comparison with the equivalent arc approximation approach is given to justify the amount of improvement achieved in calculation of cross sectional properties by employing the proposed approach. This is followed by sensitivity analysis based on the discretization settings such as STL exporting tolerance and grid size.

\subsubsection{Case 1: asymmetrical non-uniform flat end mill}

Cutting tools with two flutes are known as asymmetrical tools in terms of cross sectional properties around principal axis $\mathrm{x}$ and $y$. Even for flat end mills, the cross-sectional properties may vary due to variable flute geometry. An asymmetrical milling tool with two flutes and varying flute geometry is considered in this case, where the basic dimensions are given in Fig. 4a. The cross section obtained from the exact CAD model and the cross section drawn as arc approximation are shown in Fig. 4b, as well.

The cross-sectional properties at $\mathrm{z}=5 \mathrm{~mm}$ level are calculated by exact CAD model, the proposed STL slicing method and the arc approximation method, where the results are compared in Fig. 5. The moment of inertia is calculated around feed and cross feed axis, including the effect of helix angle. However, for any reason, if the effect of helix angle needs to be removed, it can be done during the calculation if inertia by rotating the principal axis by the lag angle due to the helix angle on the cutting flutes. The parameters required for the arc approximation are measured from

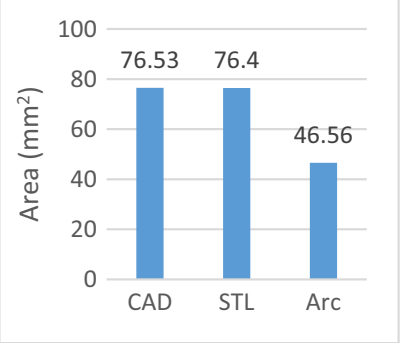

(a) Area

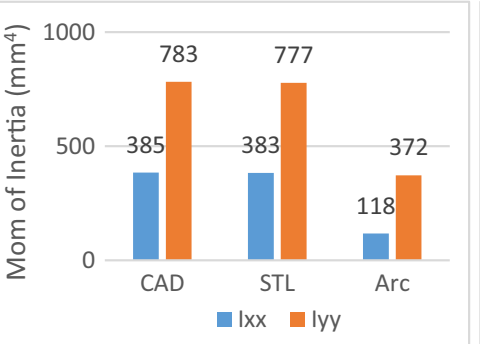

(b) Inertia

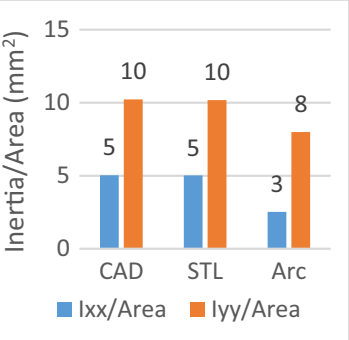

(c) Inertia to area ratio 
Fig. 6 Variation of cross sectional properties in case 1
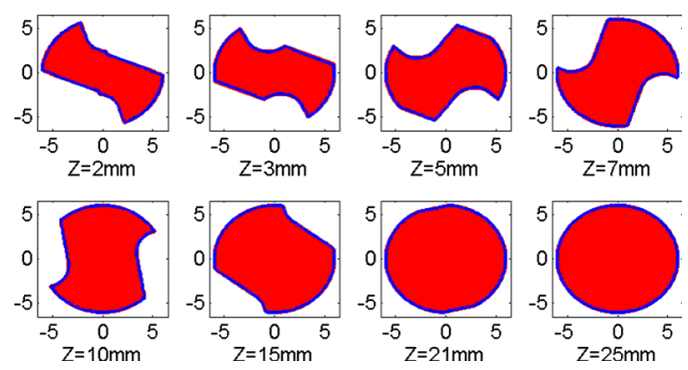

(a) Cross section geometries along tool axis.
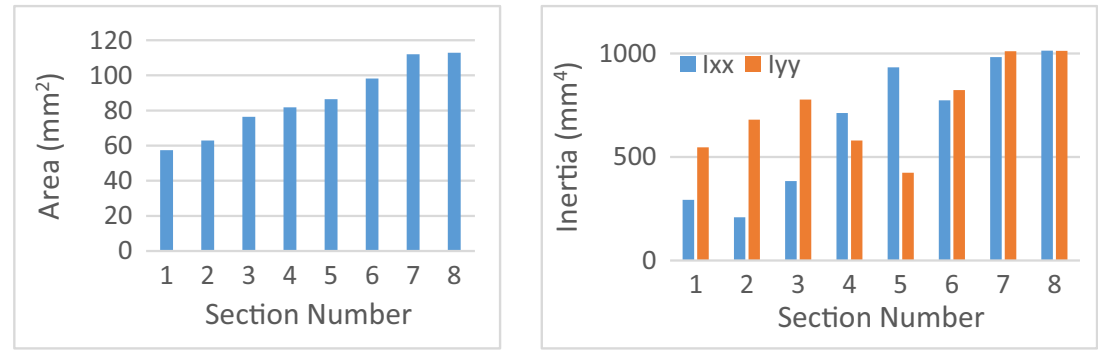

(b) Cross sectional properties

CAD as gash depth, $f_{\mathrm{d}}=3.53 \mathrm{~mm}$, eccentricity, $a=1.76 \mathrm{~mm}$, and arc radius, $r=4.235 \mathrm{~mm}$.

In Fig. 5a, it is seen that arc approximation incurs about $40 \%$ error in area calculation, where the error is around 70 and $50 \%$ in calculation of moment of inertias around $x$ and $y$ axis, respectively (see Fig. 5b). Considering that the structural response of the tool is related to the ratio between inertia and area it is noteworthy to compare the inertia-to-area ratio as shown in Fig. 5c. It is again observed that arc approximation approach incurs around 40 and $60 \%$ error for $\mathrm{Ixx} / \mathrm{A}$ and Iyy/A, respectively. However, the proposed STL slicing algorithm decreases the error down to $0.5 \%$ in all the cases.

The cross sections along the tool axis are shown for eight levels in Fig. 6a, where it is clearly seen that the cross section geometry varies significantly at different $z$ levels. The crosssectional properties calculated by the proposed STL slicing approach are plotted in Fig. 6b, which quantitatively shows the variation in the sectional properties. The cross-sectional area increases along the tool axis, where the moment of inertia shows controversial variations with respect to each other due to the asymmetry in the tool sections.

\subsubsection{Case 2: symmetrical uniform flat end mill}

In case 2, the proposed approach is applied on a symmetrical flat end mill with uniform cross section along tool axis, where the accuracy of the calculations is compared with exact CAD measurements and the arc approximation approach. The cutting tool geometry is shown in Fig. 7a. The cross section geometry obtained by arc approximation and the exact $\mathrm{CAD}$ are illustrated in Fig. 7b. The parameters required for arc approximation are measured from $\mathrm{CAD}$ as gash depth, $f$ $\mathrm{d}=1.45 \mathrm{~mm}$, eccentricity, $a=1.30 \mathrm{~mm}$, and arc radius, $r=4.72 \mathrm{~mm}$.

The cross-sectional properties measured from exact CAD model, calculated through STL slicing and arc approximation are plotted in Fig. 8a, b, where the inertia-to-area ratio is plotted in Fig. 8c.

In Fig. 8a, b, it is seen that the STL slicing algorithm calculates the area and inertia very close to the exact CAD measurements, where the error is $0.22 \%$ for area and $0.66 \%$ for inertia. The error incurred by the arc approximation reaches up to $13 \%$ for area and $19 \%$ for inertia even though the arc approximation parameters are measured from the CAD model, which is the best case. As the ratio of inertia to area is
Fig. 7 Flat end mill considered in case 2

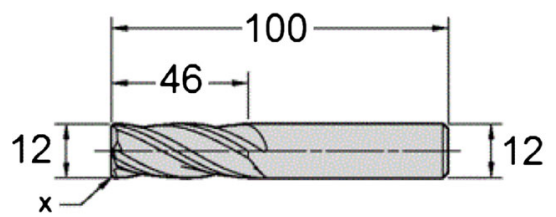

(a) Tool geometry

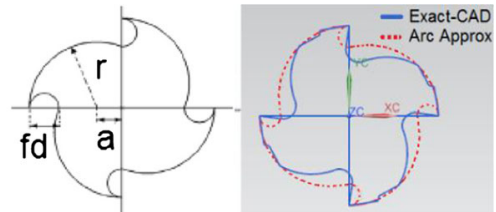

(b) Arc approximation \& Exact-CAD 
Fig. 8 Comparison of calculated section properties

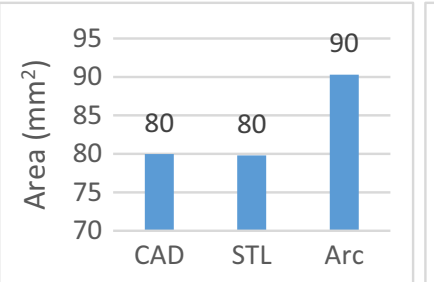

(a) Area

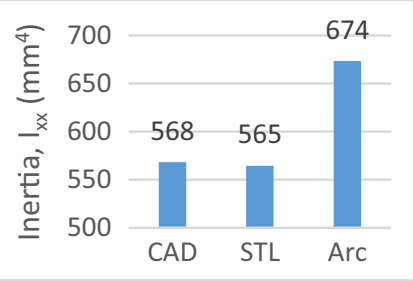

(b) Inertia

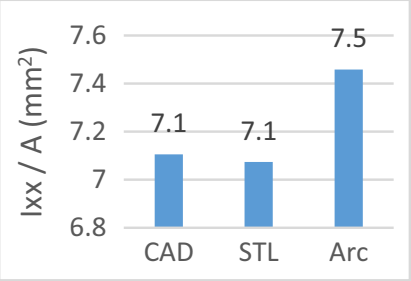

(c) Inertia to area ratio considered, the incurred errors are 0.4 and 5\% for STL slicing algorithm and arc approximation algorithm, respectively. Considering the improvements in the incurred error even for the best arc approximation of a simple end mill case it can be said that the proposed approach will minimize the geometrical modeling errors and improve the generality of RCSA method.

\subsubsection{Case 3: symmetrical taper ball end mill}

Taper ball end mills are widely used in aerospace industry for machining of integrally bladed turbine disks. In case 2 , the proposed STL slicing algorithm is applied on calculation of cross-sectional properties of a taper ball end mill. The cutting tool is illustrated in Fig. 9a, where the tool has four cutting flutes with helix angle of $38^{\circ}$ and taper angle of $4^{\circ}$, the tool tip diameter is $6 \mathrm{~mm}$, and shank diameter is $12 \mathrm{~mm}$. The variation of the tool section at eight levels along the tool axis is given in Fig. 9b, as well. It is seen that the cross section geometry is varying significantly along the tool axis. In such circumstances, the parameters required for arc approximation need to be measured for each section under interest, which may be impractical, inaccurate or very time consuming. Thus, STL slicing would serve as a more practical and accurate way of calculating the cross-sectional properties for general tool geometries.

The variation of area and moment of inertia around $x$ axis at these eight levels are given in Fig. 10 together with the incurred error for different accuracy settings such as grid size and STL export tolerance. The moment of inertia includes the effect of helix angle, as well. In this case, the tool has equally spaced four cutting flutes and hence it has symmetrical cross- sectional properties with respect to feed and cross feed axis, i.e., $x$ and $y$.

The cross-sectional properties, calculated for grid size and STL tolerance of $0.02 \mathrm{~mm}$, are plotted in Fig. 10a, b, respectively. The calculation time, for the mentioned accuracy settings, is less than $0.15 \mathrm{~s}$ per cross section. It is seen that both area and inertia is increasing nonlinearly along the tool axis. This is reflected to the ratio of inertia to area as shown in Fig. 10c, which is increasing from 0.5 to $9 \mathrm{~mm}^{2}$. The incurred error is compared for different accuracy settings of grid size and STL tolerance, to see practical values for such accuracy settings. The effect of grid size on the accuracy of area calculation and inertia calculation is given in Fig. 10d, e, respectively. It is seen that when grid size is less than $0.3 \mathrm{~mm}$, the incurred error is at most $1 \%$ for all of the sections except the first section, i.e., almost the tip of the tool, having a very small area. However, this can still be improved by employing adaptive selection of grid size for small sections. The effect of STL tolerance on calculation of area and inertia is plotted in Fig. 10f, g. The error is at most $\% 1$ for the cases where STL tolerance is less than $0.5 \mathrm{~mm}$, which is a practical tolerance value for exporting STL of such tool models.

In this section, the proposed STL slicing algorithm is applied on three different types of cutting tool to demonstrate the potential benefits and improvements achieved in geometrical modeling of generalized cutting tools. According to the results it can be concluded that the proposed approach can accurately capture the cross-sectional variations along the tool axis and as well as the asymmetry of the section. That would lead to more accurate structural modeling of cutting tools for simulation of static deflection and dynamic response. In the next section, the Timoshenko
Fig. 9 Taper ball end mill considered in the case study
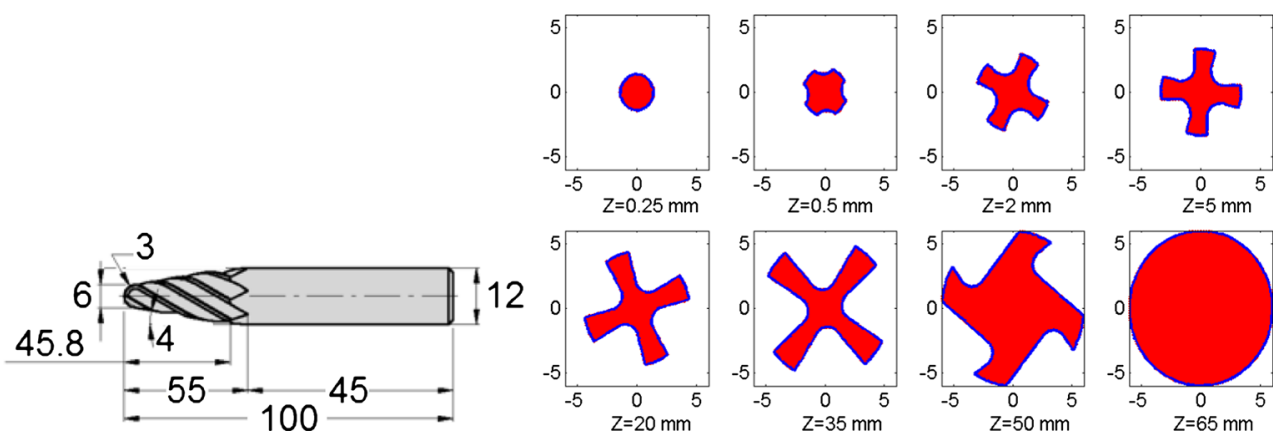

(a) Tool geometry
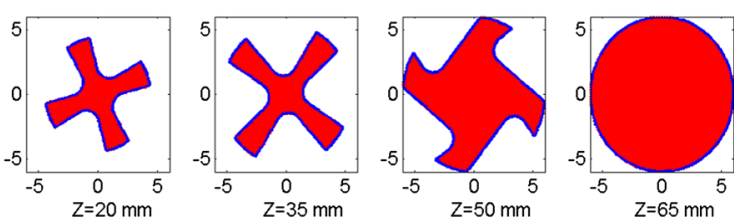

(b) Cross section geometries at several axial levels 
Fig. 10 Variation of cross section properties and incurred error

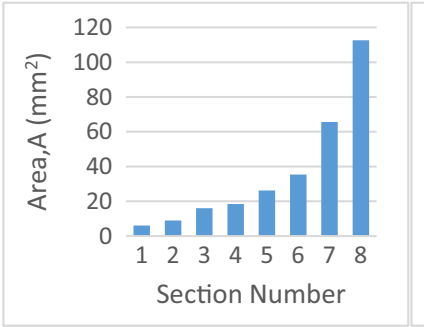

(a) Area

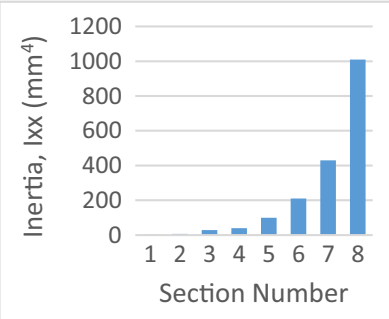

(b) Inertia

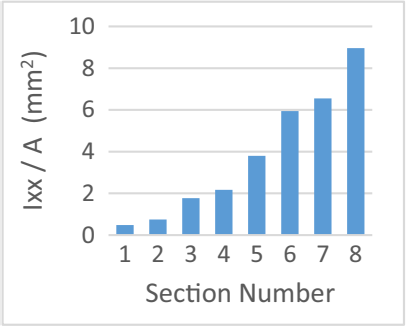

(c) Inertia -to - area ratio

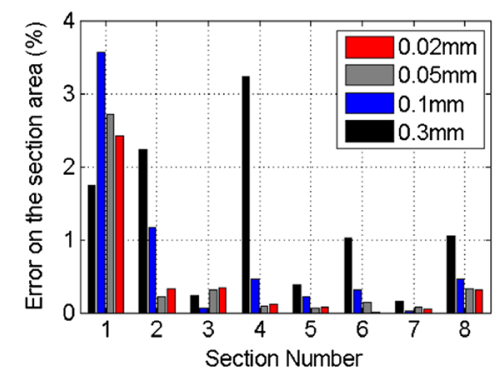

(d) Effect of grid size on area error

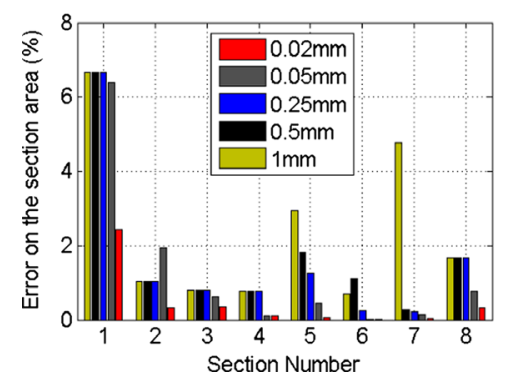

(f) Effect of STL accuracy on area error

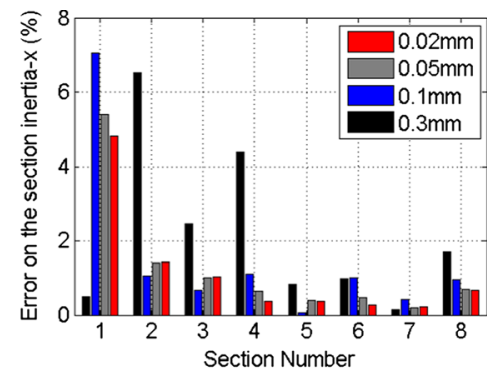

(e) Effect of grid size on inertia error

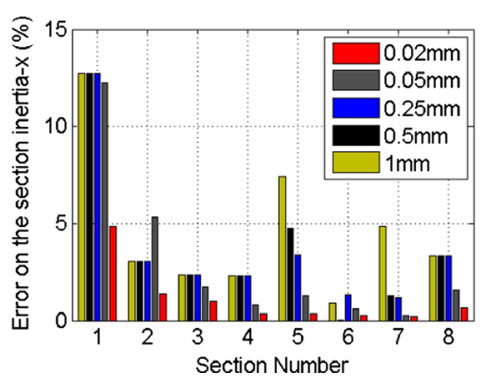

(g) Effect of STL accuracy size on inertia error beam model based on the solution given by AristizabalOchoa [4] for prediction of free-free response of the cutting tool is presented briefly.

\section{Analytical prediction of free-free dynamic response of the tool}

In this paper, the tool body is modeled as multisegmented Timoshenko beam. The insufficiency of Euler-Bernoulli beam has been emphasized previously by comparing the results obtained by Timoshenko beam model with those of Euler-Bernoulli, where a considerable amount of improvement has been observed in the predictions [11]. In prediction of the free-free response of the tool, the tool body is divided into $\boldsymbol{m}$ number of segments, where the first segment is the shank of the tool, and then the fluted section is rigidly coupled to the tool shank segment by segment as illustrated in Fig. 11.

In application of the proposed method, selection of the segment lengths is quite important for accuracy of the results and computational performance. For the cases, where the cross section of the fluted portion is significantly varying along the tool axis, the segment lengths should be selected considering the variation. However, if the fluted cross section is not that variable, the segment lengths should be selected as long as possible to improve calculation performance.

The characteristic equation resulting from the eigenvalue problem of the m-segmented Timoshenko beam is expressed as a $4 \mathrm{~m} \times 4 \mathrm{~m}$ matrix. Although the elements of such a matrix represent physical quantities the condition number of such large order matrix may become very large as the elements are highly nonlinear. Thus, accurate identification of higher roots of the determinant function becomes almost impossible. Such a complication is handled by solving the eigenvalue problem for a uniform, single-segment free-free Timoshenko beam as proposed by Erturk et al. [11]. Then, the free-free beams of different segments are coupled by using the end point FRFs to obtain the multi-segmented cutting tool.

In this study, the frequency and mode shape equations of uniform Timoshenko beam with generalized end conditions presented by Aristizabal-Ochoa [4] is utilized, where classical Eigen solution is used. In this section, the formulation of the utilized Timoshenko beam solution is briefly given together 


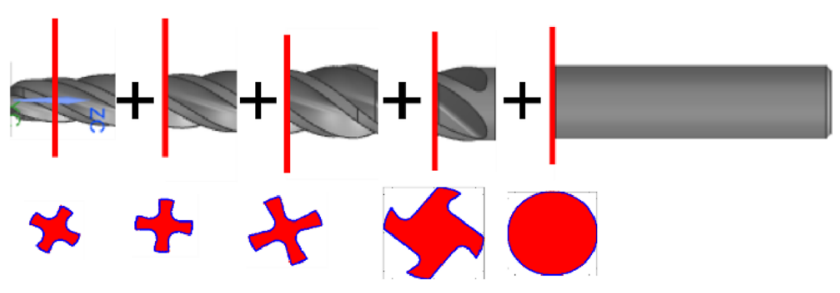

Fig. 11 Rigid coupling of tool segments

with the search algorithm proposed for obtaining the roots of the characteristic equation. Then, the results obtained by accurate modeling of tool cross section through STL slicing proposed in this paper are compared with those obtained by arc approximation of the tool cross section.

\subsection{Timoshenko beam model of tool segments}

In this study, the Timoshenko beam solution for generalized end conditions is simplified to represent free-free end conditions. Although the use of the simplified solution has been shown in simulation of free-free tool response, the formulation is given for completeness of the solution and to explain the iterative numerical approach proposed to solve the characteristic equation in Section 4.1.1. So that, some of the terms in the general solution is eliminated and the simplified form of the characteristic equation is written as follows:

$\left|\begin{array}{ll}D_{11} & D_{12} \\ D_{21} & D_{22}\end{array}\right|=D_{11} D_{22}-D_{12} D_{21}=0$

The terms in the characteristic Eq. (6) are written as follows:

$D_{11}=(\alpha-\lambda)(\cos \alpha-\cosh \beta)$

$D_{12}=(\lambda-\alpha) \sin \alpha+\frac{\lambda \alpha}{\beta \delta}(\beta-\delta) \sinh \beta$

$D_{21}=-\lambda \alpha \sin \alpha+\frac{\alpha-\lambda}{\delta-\beta} \beta \delta \sinh \beta$

$D_{22}=\lambda \alpha(\cosh \beta-\cos \alpha)$

In above expressions, $\alpha$ and $\beta$ are the dimensionless frequency numbers written in terms of geometrical and material properties of the beam section:

$\alpha=\sqrt{\Omega+\varepsilon}, \beta=\sqrt{\varepsilon-\Omega}$

where,

$$
\begin{aligned}
& \Omega=\frac{b^{2}\left(s^{2}+R^{2}\right)}{2}, \varepsilon=b \sqrt{\frac{1}{4} b^{2}\left(s^{2}+R^{2}\right)^{2}-\left(b^{2} s^{2} R^{2}-1\right)}, \\
& b^{2}=\left(\frac{\rho A \omega^{2} L^{4}}{E I}\right), s^{2}=\frac{E I}{k^{\prime} A G L^{2}}, R^{2}=\frac{I}{A L^{2}}
\end{aligned}
$$

In Eq. (12), $\rho, E, G, k^{\prime}, A, I$, and $L$ represent the density, Young's modulus, shear modulus, shear coefficient, crosssectional area, cross-sectional area moment of inertia, and length of the segment, respectively. For a hollow circular cross section, the shear coefficient, $k$, can be written in terms of the poison's ratio and the ratio of inner diameter to the outer diameter as mentioned by Blevins [21]. This is valid even for the shank segment of the tools with internal cooling hole. If the cutting tool includes an internal cooling hole, its diameter may be either known prior or numerically calculated from the STL cross section.

$k^{\prime}=\frac{6(1+v)\left(1+m^{2}\right)^{2}}{(7+6 v)\left(1+m^{2}\right)^{2}+(20+12 v) m^{2}}$

where,

$m=\frac{d_{\text {inner }}}{d_{\text {outer }}}$

The dimensionless frequency numbers, $\alpha_{r}$ and $\beta_{r}$, and the natural frequency corresponding to $r$ th mode are obtained by solving the characteristic Eq. (1). Where the iterative solution algorithm to obtain the natural frequencies from the characteristic Eq. (1) is detailed in the following section. Then, the dimensionless frequency numbers are used together with the geometrical parameters given through Eqs. (11) and (12) to derive the mode shape expressions for the dynamic transverse deflection, $\varnothing_{r}(x)$, and dynamic bending rotation, $\psi_{r}(x)$, as follows:

$\varnothing_{r}(x)=A_{r}\left[C_{1} \sin \left(\frac{\alpha_{r}}{L} x\right)+C_{2} \cos \left(\frac{\alpha_{r}}{L} x\right)+C_{3} \sinh \left(\frac{\beta_{r}}{L} x\right)+C_{4} \cosh \left(\frac{\beta_{r}}{L} x\right)\right]$

$\psi_{r}(x)=\frac{A_{r}}{L}\left[\lambda_{r}\left(C_{1} \cos \left(\frac{\alpha_{r}}{L} x\right)-C_{2} \sin \left(\frac{\alpha_{r}}{L} x\right)\right)+\delta_{r}\left(C_{3} \cosh \left(\frac{\beta_{r}}{L} x\right)+C_{4} \sinh \left(\frac{\beta_{r}}{L} x\right)\right)\right]$ 
In Eqs. (14) and (15) the following definitions are used:

$$
\begin{aligned}
\lambda_{r} & =\alpha_{r}-\frac{b^{2} s^{2}}{\alpha_{r}}, \delta_{r}=\beta_{r}+\frac{b^{2} s^{2}}{\beta_{r}} \\
C_{1} & =\mathrm{L}, C_{2}=-\frac{D_{11}}{D_{12}} C_{1}, C_{3}=\frac{\alpha_{r}-\lambda_{r}}{\delta_{r}-\beta_{r}} C_{1}, C_{4} \\
& =\frac{\lambda_{r} \alpha_{r}}{\beta_{r} \delta_{r}} C_{2},(r=1,2,3, \ldots)
\end{aligned}
$$

Finally, $A_{r}$ is a constant obtained by the mass normalization of a the eigenfunctions such that the below orthogonality condition is satisfied;

$$
\int_{x=1}^{L}\left\{U_{r}(x)\right\}^{T}[M]\left\{U_{s}(x)\right\} d x=\left\{\begin{array}{c}
1, r=s \\
0, r \neq s
\end{array}\right.
$$

where.

$$
\left\{U_{r}(x)\right\}=\left\{\begin{array}{l}
\varnothing_{r}(x) \\
\psi_{r}(x)
\end{array}\right\}, \quad[M]=\left[\begin{array}{cc}
\rho A & 0 \\
0 & \rho I
\end{array}\right]
$$

In addition to the flexible body vibration, the tool body has two rigid body modes under free end conditions, where the corresponding Eigen functions $\phi_{0}^{t}(x)$ and $\phi_{0}^{r}(x)$ are written as below:

$\phi_{0}^{t}(x)=\sqrt{\frac{1}{\rho A L}}$

$\phi_{0}^{r}(x)=\sqrt{\frac{1}{\rho A L^{3}}}\left(x-\frac{L}{2}\right)$

Once the Eigen functions are written the receptance functions those relate the linear and angular displacements to the forces and

After Algorithm 1 runs, the whole frequency range is portioned into sub regions where natural frequencies exist as shown in Fig. 12b. Then, the natural frequencies are precisely identified by Algorithm 2, through golden section moments, i.e., $H, N, L$, and $P$ are calculated considering the sufficient number of modes. The expressions for the receptance functions are given in reference. After all of the end point FRFs of a sub segment are calculated, the free-free sub segments are coupled together, as described in Section 2, in order to obtain the free-free FRF of the overall tool body.

\subsubsection{Iterative solution procedure for the reduced characteristic equation}

The reduced characteristic equation (6) is a highly nonlinear equation, thus analytical solution is not available. In this study, the characteristic equation is rather solved by a two-pass iterative algorithm in order to obtain the natural frequencies. In the first pass, the frequency regions where the natural frequencies may exist are identified. Then, in the second pass, the natural frequencies at each region are iteratively calculated by using golden section search algorithm.

The variation of the characteristic equation with frequency for a carbide beam with $100 \mathrm{~mm}$ of length and $10 \mathrm{~mm}$ of diameter, where $E=650 \mathrm{GPa}, G=243 \mathrm{GPa}, \rho=14,500 \mathrm{~kg} / \mathrm{m}^{3}$, and $v=0.3$, is plotted in Fig. 12 .

The equation is calculated for a frequency range of $1 \mathrm{~Hz}$ to $250 \mathrm{KHz}$. A frequency value is said to be the root of the characteristic equation, i.e., the natural frequency, when it yields the equation to be zero. In other words, the frequency values at which the function plot crosses $y=0$ line are the natural frequencies. As the full-scale function plot (see Fig. 12a) is observed, it is seen that the function cross $y=0$ line at some frequency values. Once the plot is zoomed for $y \pm 1$ range the crossings become even clearer. The iterative algorithm proposed in this paper finds the natural frequencies based on this information.

search in each frequency range. In Fig. 12c, a sub region between 2000 and $10,000 \mathrm{~Hz}$ is shown, where the natural frequency is around $6000 \mathrm{~Hz}$. In the golden section search, the sign change of the function is tracked until the frequency range converges within $1 \mathrm{~Hz}$.

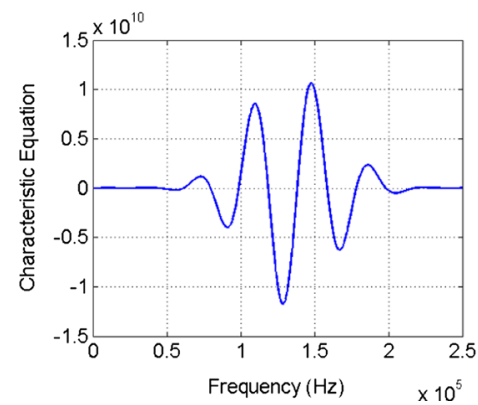

(a) full scale

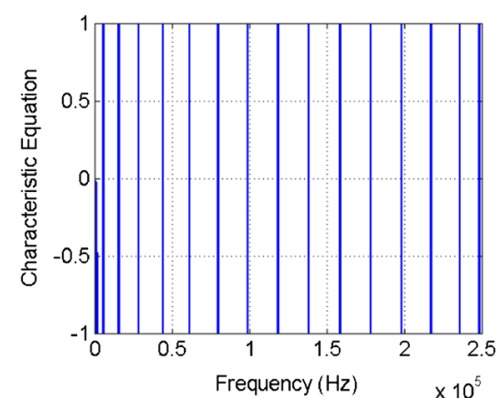

(b) zoomed in y axis

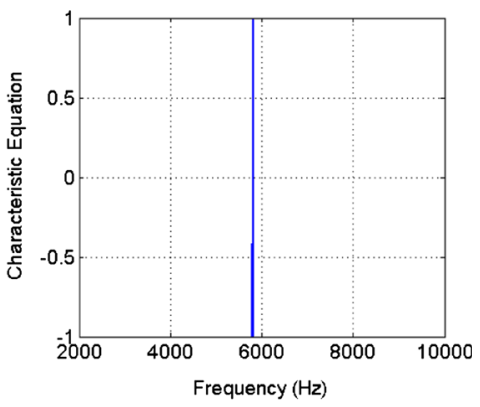

(c) root finding

Fig. 12 Plot of the characteristic equation for a representative case 


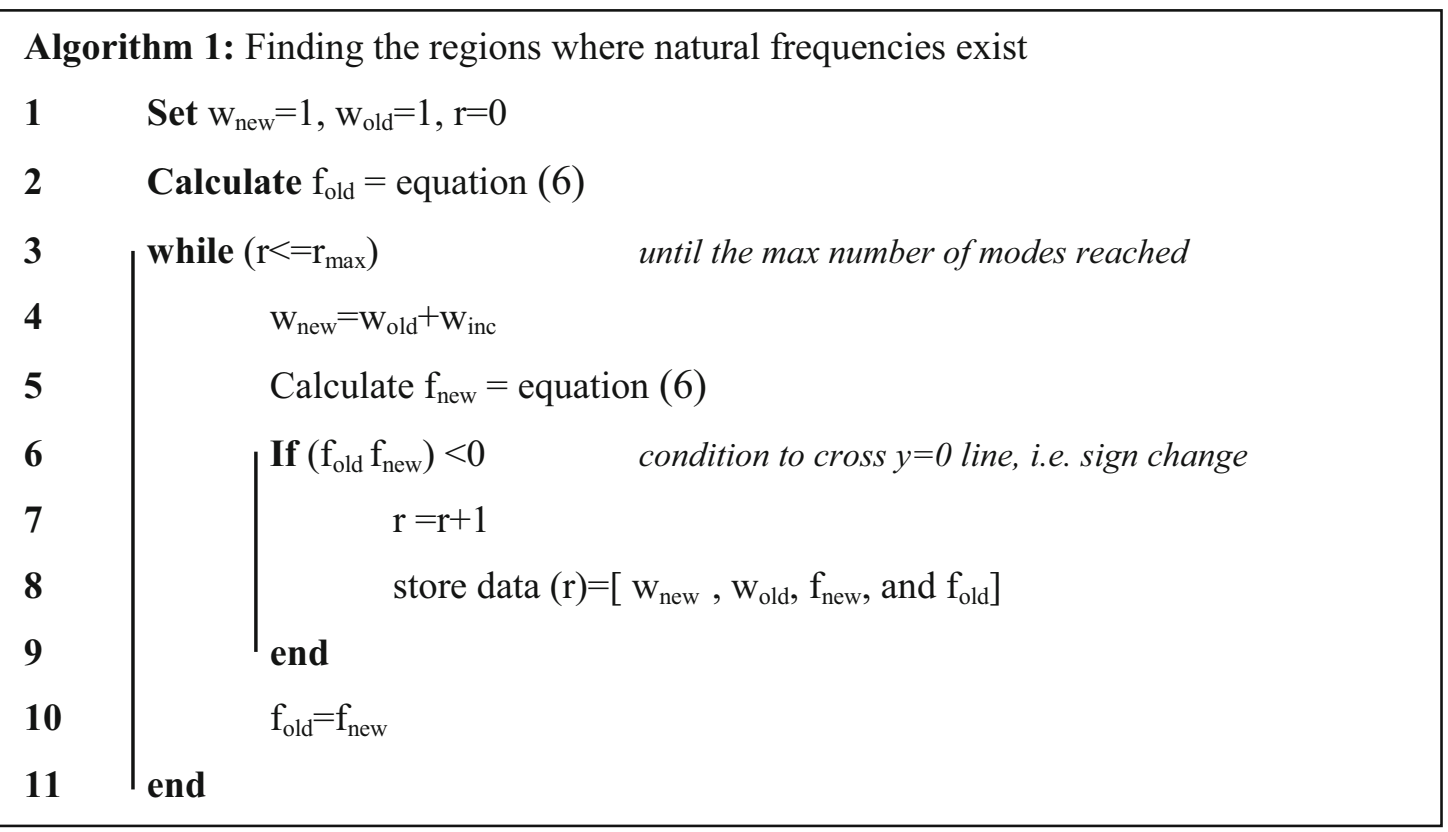

In this section, the analytical modeling of free-free receptance of the cutting tool through Timoshenko beam approach is discussed. The cutting tool is modeled as combination of uniform Timoshenko beam segments. Then, the receptance of each sub segment is coupled in order to obtain the free-free response of the whole cutting tool body. The natural frequencies are identified by numerical solution of the reduced characteristic equation.

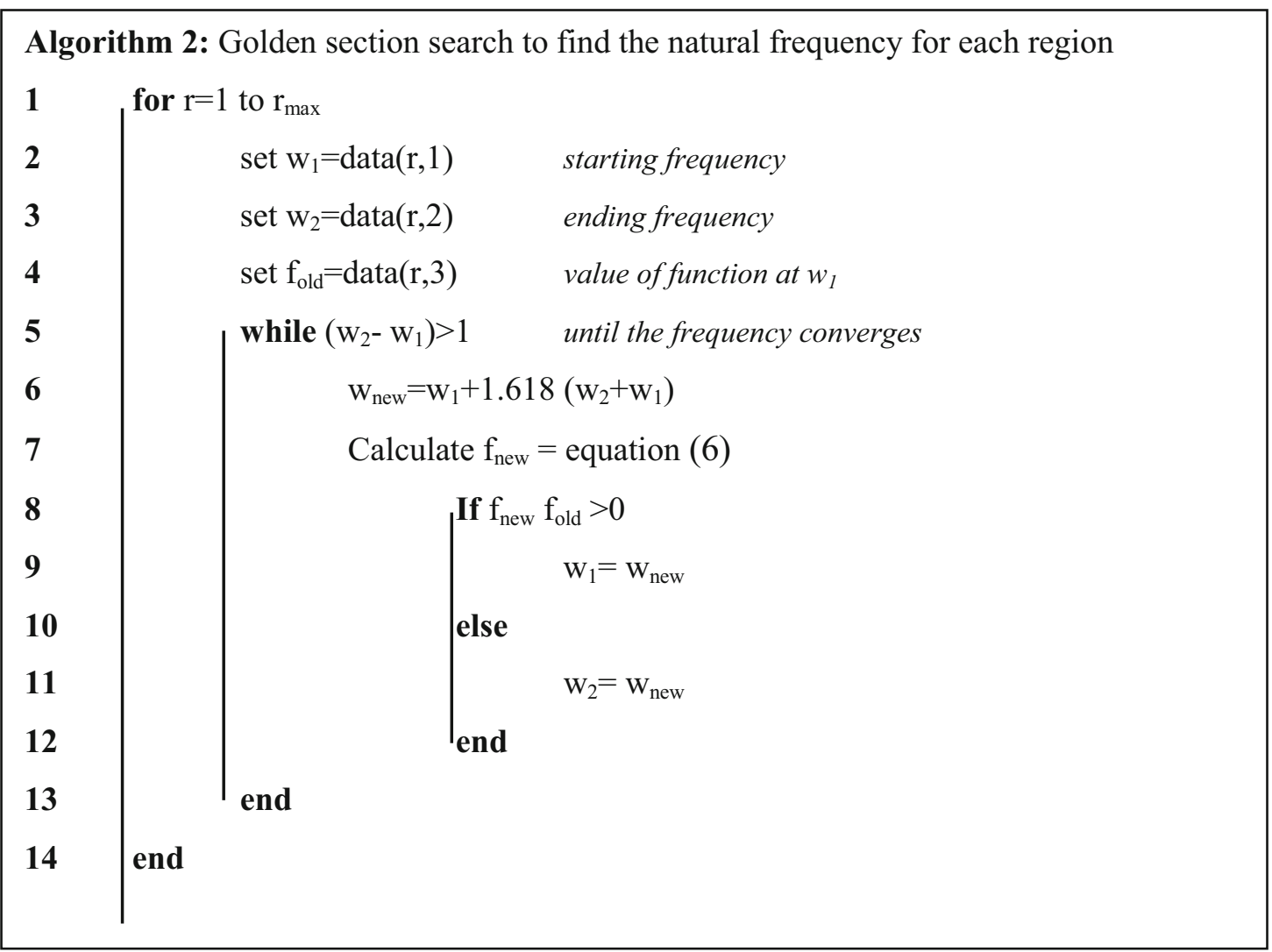




\subsection{Simulation of free-free tool response}

In this section, the improvement obtained by calculating the cross-sectional parameters through STL slicing algorithm is discussed. The free-free tool receptance calculated using STL slicing and arc approximation approaches are compared and conclusions are derived. The cutting tools presented in Section 3 are considered as representative cases. The material properties for carbide are used as $E=650 \mathrm{GPa}, G=243 \mathrm{GPa}$, $\rho=14,500 \mathrm{~kg} / \mathrm{m}^{3}$, and $v=0.33745$, where the material loss factor is taken as 0.01 to include damping. Simulations are performed at different tool lengths in order to cover different cases. The natural frequencies of the tool are identified from the simulated frequency response function (FRF), which is written in terms of the mode shapes, $\varnothing_{r}(x)$, modal natural frequencies, $\omega_{r}$, of the $r$ th mode, and the loss factor, $\gamma$, as follows;

$H_{11}=\sum_{r=0}^{\infty} \frac{\varnothing_{r}\left(x_{1}\right) \varnothing_{r}\left(x_{1}\right)}{(1+i \gamma) \omega_{r}^{2}-\omega^{2}}$

The rest of the transfer functions $H, L, N$, and $P$ can be found in reference [11]. Considering that the shank geometry does not change along the tool axis, in simulation of the FRF for each tool, only a single uniform cross section is used to represent the tool shank geometry, regardless of the shank length. This is another advantage of the proposed method against the FEM-based routines.

Selection of the number and axial locations of the tool segments is crucial for accuracy of the simulation freefree response. In this paper, the number and the axial locations of the segments to calculate the crosssectional properties are selected based on the variation in the cross-sectional properties. The cutting tool is considered to be composed of three main portions as the shank, the transition, and the fluted part. The shank geometry has a cylindrical cross section with a constant diameter. Thus, only one cross section is selected to represent the shank. In geometrical modeling of the transition region, the cross section at the mid location between the shank and the fluted portions is taken. In modeling of the fluted portion, for the cases with uniform cross section along the tool axis, only one section is selected similar to the shank portion. However, if the fluted portion has varying cross section additional levels are added along the tool axis based on the variation. In the simulation cases considered in this section, at most four cross sections are taken to represent the tool geometry.
Fig. 13 Comparison of the first three natural frequencies (freefree)

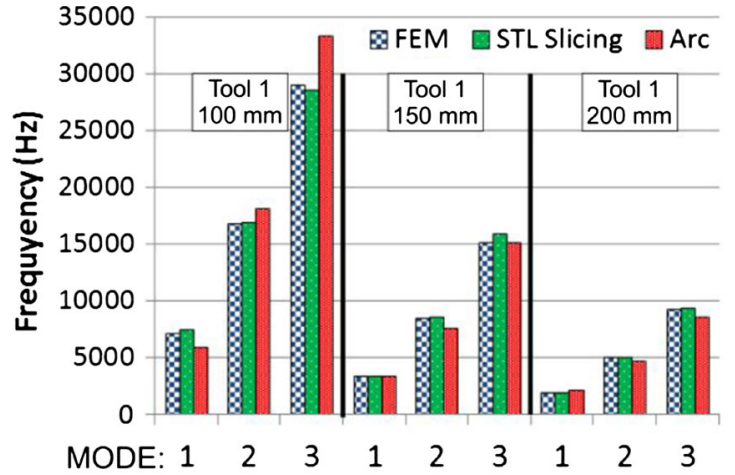

(a) Tool 1

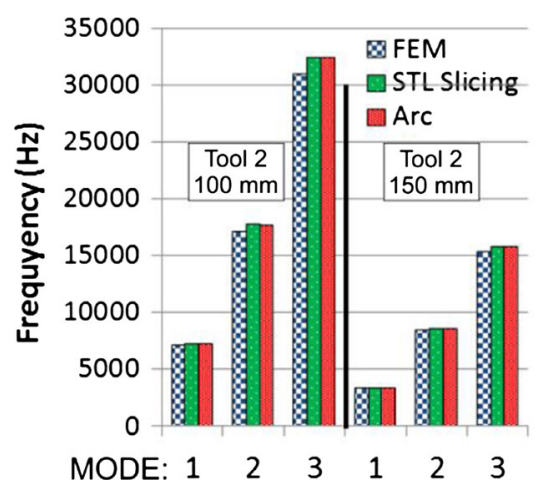

(b) Tool 2

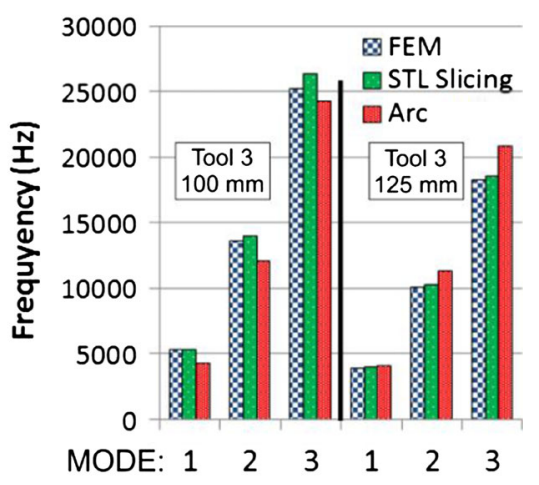

(c) Tool 3 
The natural frequencies corresponding to the first three modes, obtained by FEM, STL slicing and arc approximation approaches are plotted in Fig. 13. Here, the FEM results are taken as the baseline and the accuracy is commented with respect to the results obtained through FEM analysis. It is observed that the STL slicing algorithm predicts the natural frequency of the first three modes of Tool 1, where the fluted portion has variable cross section along the tool axis, more accurate than the arc approximation method for all cases. The results obtained for Tool 2, where the tool has uniform cross section, show that both STL slicing and arc approximation approaches perform at a similar accuracy. Finally, the results obtained for the taper ball end mill, i.e., Tool 3, show that the STL slicing method performs much better than the arc approximation method as the variation in the cross-sectional properties are handled progressively. In the comparisons, the arc approximation is used to estimate the dynamics of the taper ball end mill in such a way that the approximated crosssectional parameters are updated considering the taper angle on the cutting tool. To do so, an equivalent radius is calculated using the approximated cross-sectional parameters. Then, the equivalent radius is updated considering the taper angle. Assuming that FEM results are the most realistic, it can be said that the STL slicing approach works very well for cutting tools having variable cross sections, which will enable the receptance coupling technique to be applied on such tools. According to the simulation results, it can be said that the arc approximation method provides adequate results for the tools having uniform cross section. Based on the results presented in Fig. 13, the free-free FRF of three representative cases are plotted in Fig. 14.

In Fig. 14a, the FRF of Tool 1 at $200 \mathrm{~mm}$ length is plotted, where a significant difference between the FRF obtained by STL slicing and Arc approximation is observed in terms of both amplitude and natural frequencies. The FRF of Tool 2 at $150 \mathrm{~mm}$ is plotted in Fig. 14b, where the results obtained by both methods matches. The FRF of the taper ball end mill is given in Fig. 14c, where a significant difference between the STL slicing algorithm and arc approximation is observed.

\subsection{Comparison of simulation performance with FEM}

In this section, the performance of the proposed approach in prediction of free-free dynamic response is compared with FEM simulations for representative cases given in Table 1. In FEM techniques, it is well known that the convergence of the solution depends on the meshing quality. Thus, the effect of meshing quality on convergence is also considered for the FEM simulations. The simulation time for the case where the results converge, is taken as reference. In the FEM simulations, the convergence criterion is taken as $2 \mathrm{~Hz}$, considering the sensitivity of the tool tip FRF on the free-free response of the cutting tool body. In Table 1, the cases are given together with the FEM simulation settings together with a comparison
Fig. 14 Comparison of free-free frequency response for three cases

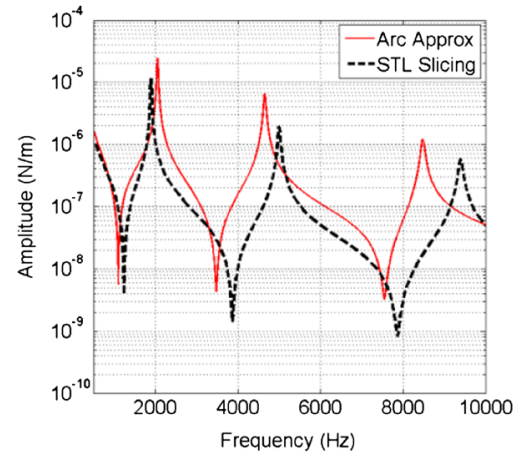

(a) Tool1-200 mm

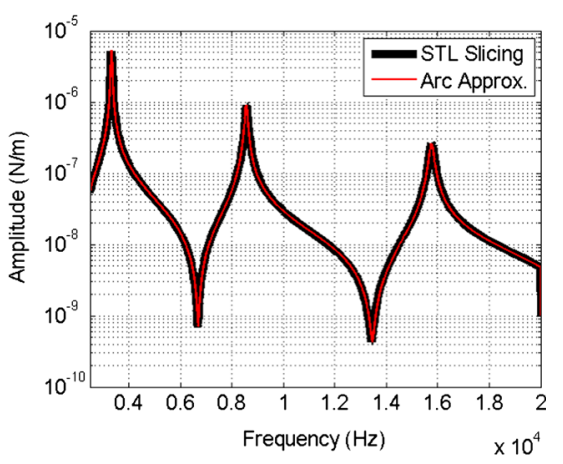

(b) Tool 2-150 mm

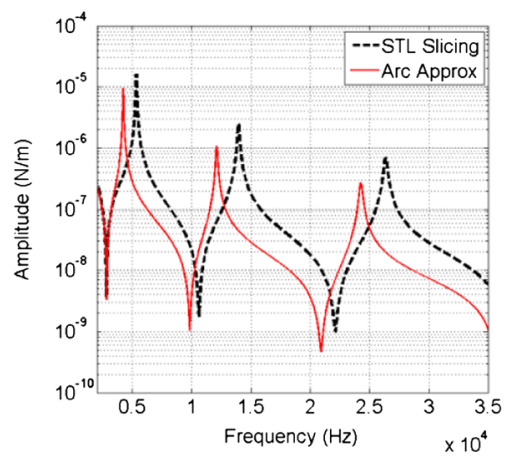

(c) Tool 3-100 mm 
Table 1 Comparison of simulation time

\begin{tabular}{|c|c|c|c|c|c|c|c|}
\hline & Mesh quality & $\begin{array}{l}\text { Number of } \\
\text { elements }\end{array}$ & Nodes & $\begin{array}{l}\text { 1 st Nat. freq. } \\
\text { FEM }(\mathrm{Hz})\end{array}$ & $\begin{array}{l}\text { Solution time- } \\
\text { FEM (s) }\end{array}$ & $\begin{array}{l}\text { Location of } \\
\text { segments }\end{array}$ & $\begin{array}{l}\text { Solution time } \\
\text { STL (s) }\end{array}$ \\
\hline \multirow{4}{*}{$\begin{array}{l}\text { Tool } 1 \\
L=100 \mathrm{~mm}\end{array}$} & Coarse & 874 & 1661 & 7485 & 3 & \multirow{4}{*}{$\begin{aligned} Z & =3 \mathrm{~mm} \\
Z & =7 \mathrm{~mm} \\
Z & =15 \mathrm{~mm} \\
Z & =25 \mathrm{~mm}\end{aligned}$} & \multirow[t]{4}{*}{1.6} \\
\hline & Medium & 5817 & 9465 & 7488 & 9 & & \\
\hline & Fine & 27,514 & 41,241 & 7488 & 70 & & \\
\hline & Extra fine & 72,949 & 112,961 & 7488 & 185 & & \\
\hline \multirow{4}{*}{$\begin{array}{l}\text { Tool } 1 \\
L=200 \mathrm{~mm}\end{array}$} & Coarse & 330 & 773 & 1893 & 3 & \multirow{4}{*}{$\begin{aligned} Z & =3 \mathrm{~mm} \\
Z & =7 \mathrm{~mm} \\
Z & =15 \mathrm{~mm} \\
Z & =25 \mathrm{~mm}\end{aligned}$} & \multirow[t]{4}{*}{1.6} \\
\hline & Medium & 1722 & 3223 & 1875 & 6 & & \\
\hline & Fine & 7636 & 12,645 & 1877 & 8 & & \\
\hline & Extra fine & 68,914 & 43,420 & 1877 & 68 & & \\
\hline \multirow{4}{*}{$\begin{array}{l}\text { Tool } 2 \\
L=100 \mathrm{~mm}\end{array}$} & Coarse & 1238 & 2319 & 7207 & 3.5 & \multirow{4}{*}{$\begin{array}{l}Z=23 \mathrm{~mm} \\
Z=49 \mathrm{~mm}\end{array}$} & \multirow[t]{4}{*}{1.85} \\
\hline & Medium & 6382 & 10,523 & 7170 & 10 & & \\
\hline & Fine & 27,441 & 41,527 & 7153 & 80 & & \\
\hline & Extra fine & 253,380 & 380,392 & 7153 & 330 & & \\
\hline \multirow{4}{*}{$\begin{array}{l}\text { Tool } 3 \\
L=125 \mathrm{~mm}\end{array}$} & Coarse & 1678 & 828 & 4702 & 3 & \multirow{4}{*}{$\begin{array}{l}Z=5 \mathrm{~mm} \\
Z=20 \mathrm{~mm} \\
Z=35 \mathrm{~mm} \\
Z=55 \mathrm{~mm}\end{array}$} & \multirow[t]{4}{*}{4.2} \\
\hline & Medium & 4316 & 7504 & 4327 & 9 & & \\
\hline & Fine & 18,050 & 28,434 & 4291 & 90 & & \\
\hline & Extra fine & 106,721 & 162,985 & 4289 & 661 & & \\
\hline
\end{tabular}

of FEM simulation time and STL slicing solution time. The simulation time corresponding to the converged case is given in bold characters. As the converged simulation times are compared, it is seen that there is at least approximately four times time saving in case 2, where the free-free dynamics of Tool 1 is simulated. This difference drastically increases for the tools having variable cross section. For instance, the time saving is more than 20 times for Tool 2 and Tool 3, where Tool 3 is a tapered ball-end mill.

\section{Experimental verification}

The proposed approach is demonstrated on prediction of tool tip FRF in robotic milling setup, which has a spindle with HSK A63 connection interface, where the tool holder is hydraulic type. The 3D models of the tools used in the experimental verification phase were obtained from SANDVIK Coromant (C) under a non-disclosure agreement. Thus, the either the geometrical details or the 3D models of the tools could not be given explicitly. In the first case, the tool tip FRF of an 18-mm end mill with 2 cutting flutes in $-\mathrm{x}$ and $-\mathrm{y}$ directions, is predicted. The proposed approach is applied on prediction of tool tip dynamics for $10 \mathrm{~mm}$ end mill with 4 cutting flutes and a 4fluted taper ball end mill having $16 \mathrm{~mm}$ of shank diameter in case 2 and case 3 , respectively. In these cases, the connection parameters such as linear stiffness, linear damping, rotational stiffness, and rotational damping are identified only in $-x$ direction, where a blank geometry having the same diameter with the tool shank is used. Henceforth, the details of the identification of connection parameters are given in the next section, which is followed by the simulation and experimental results. In the FRF simulations, $2 \%$ of material damping is assumed. The drawings of the tools cannot be provided due to the confidentiality issues agreed with the tool manufacturer.

\subsection{Identification of connection parameters}

Prediction of tool tip dynamics through receptance coupling requires the connection parameters as briefly explained in Section 2. These parameters depend on the clamping torque and the length of the tool shank inside the collet. In the experimental verifications, a hydraulic type tool holder is used, where the clamping mechanism is driven by a screw providing repeatability in clamping. Thus, the clamping torque is assumed to stay the same after several clamping and unclamping the same
Table 2 Identified connection parameters for the experimental cases

\begin{tabular}{lllllll}
\hline & $\begin{array}{l}\text { Blank length } \\
(\mathrm{mm})\end{array}$ & $\begin{array}{l}\text { Lcontact } \\
(\mathrm{mm})\end{array}$ & $\begin{array}{l}k_{\mathrm{x}} \\
(\mathrm{N} / \mathrm{m})\end{array}$ & $\begin{array}{l}k_{\theta \mathrm{x}} \\
(\mathrm{Nm} / \mathrm{rad})\end{array}$ & $\begin{array}{l}c_{\mathrm{x}} \\
(\mathrm{Ns} / \mathrm{m})\end{array}$ & $\begin{array}{l}c_{\theta \mathrm{x}} \\
(\mathrm{Nms} / \mathrm{rad})\end{array}$ \\
\hline D10 Blank & 50 & 20 & $8.950 \mathrm{e} 6$ & $2.91 \mathrm{e} 4$ & 65 & 0.82 \\
D12 Blank & 110 & 20 & $13.95 \mathrm{e} 6$ & $8.97 \mathrm{e} 4$ & 135 & 0.95 \\
D18 Blank & 75 & 35 & $20.9 \mathrm{e} 6$ & $30.5 \mathrm{e} 4$ & 45 & 5.00 \\
\hline
\end{tabular}


tool to the tool holder. This assumption is verified by performing the impact hammer test three times on the $18-\mathrm{mm}$ diameter blank, where the hydraulic tool holder is untightened and then tightened back before each impact hammer test. In identification of the connection parameters, the nonlinear least square optimization function of MATLAB $\odot$ is used. The linear stiffness, linear damping, rotational stiffness, and rotational damping parameters are used are variables to minimize the least square error between the measured and predicted FRF within the frequency region under interest. The identified connection parameters in $X$ direction are given in Table 2.

The FRF at the tip of the tool holder, i.e., G33, is plotted in Fig. 15a, where low frequency modes, due to the robotic platform, are clearly observed. The three FRF measurements at the tip of $18 \mathrm{~mm}$ diameter tool blank are plotted in Fig. $15 \mathrm{~b}$, where the predicted FRF using the identified connection parameters are plotted, as well. The connection parameters are then used to predict the FRFs in $Y$ direction as a first phase verification. The predicted FRF in $Y$ direction is plotted together with the measurements in Fig. 15c.
In Fig. 15b, it is seen that the measured FRFs do not significantly vary both in terms of frequency and amplitude, which confirms that the constant clamping torque assumption is reasonable. The comparison of the predicted FRF with the three FRF measurements show that the identified connection parameters work well for the predictions. In Fig. 15b, a reasonable agreement is observed between the predictions and measurements in $Y$ direction FRF, where the predictions are performed using the connection parameters obtained in $X$ direction. Similarly, the predicted and measured FRFs in $X$ and $Y$ directions for 16- and 10-mm diameter blanks are plotted in Fig. 15d-g, respectively, where good agreement is observed.

\subsection{8-mm diameter end mill}

In the first case, the FRF of a two-fluted 18-mm diameter end mill is predicted, which represents a non-uniform tooling case. The product code of the tool is Sandvik 2P232-1800-NA H10F. The connection parameters obtained using the $18-\mathrm{mm}$ diameter blank are used in the predictions. The tool has

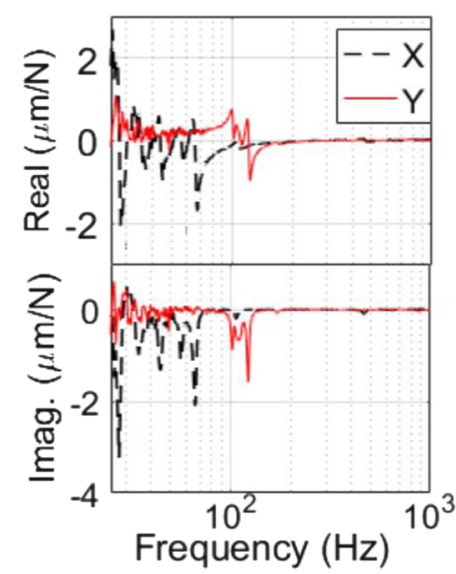

(a) FRF of the robotic platform
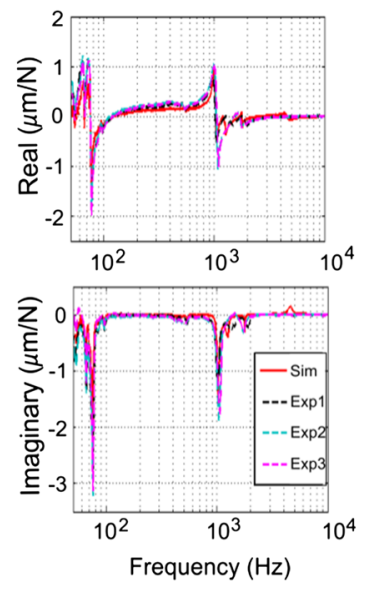

(b) D18 Blank - Gxx
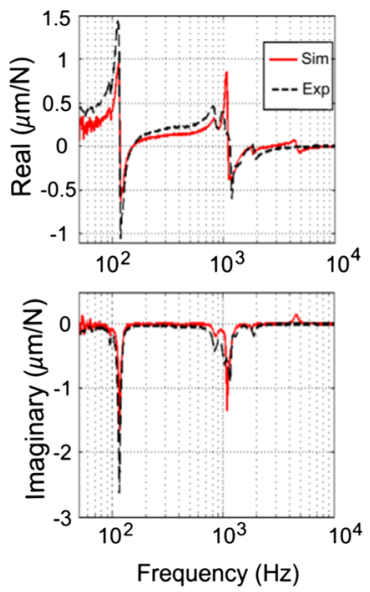

(c) D18 Blank - Gyy

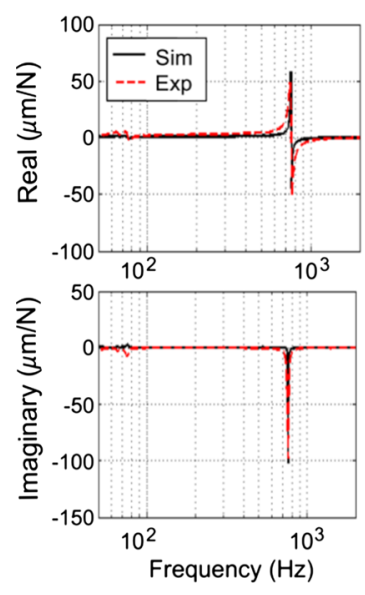

(d) D12 Blank - Gxx

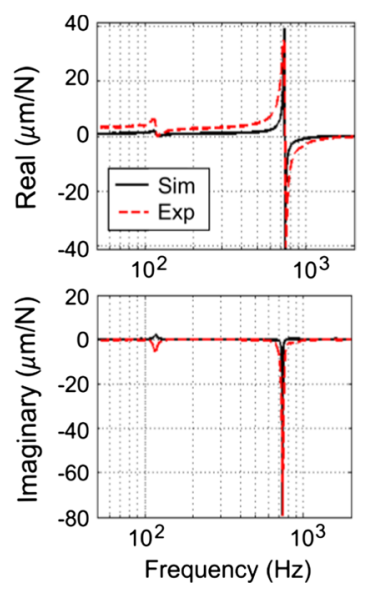

(e) D12 Blank - Gyy

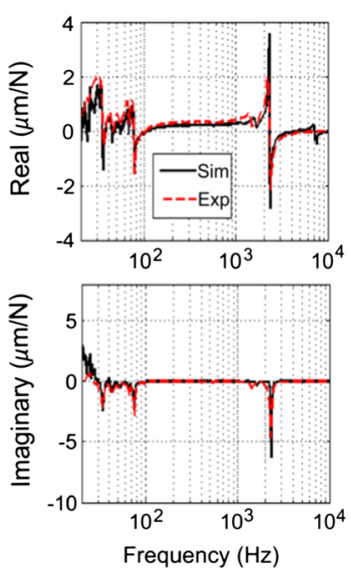

(f) D10 Blank - Gxx
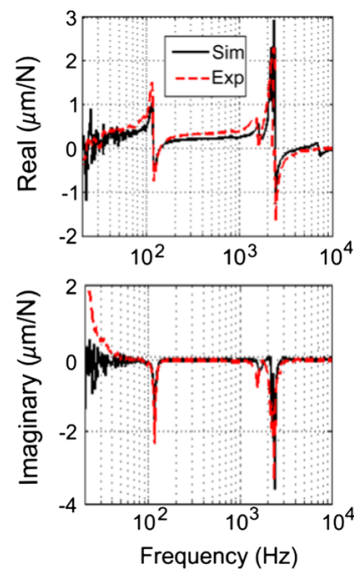

(g) D10 Blank - Gyy

Fig. 15 Measured and predicted FRFs at the tip of 18-mm diameter blank 
$105 \mathrm{~mm}$ overhang length, where length of the shank section inside the tool holder is $35 \mathrm{~mm}$. The FRFs are predicted in $X$ and $Y$ directions, which are then verified by experimental results as plotted in Fig. 16, where a good agreement is observed in terms of both the natural frequencies and the amplitudes. The low frequency mode contributed by the robotic platform is predicted and measured at 75 and $117 \mathrm{~Hz}$ in $X$ and $Y$ directions, respectively. The first mode contributed by the cutting tool is observed around $960 \mathrm{~Hz}$ in $X$ direction and $1012 \mathrm{~Hz}$ in $Y$ direction, which are predicted almost perfect. The second mode, which is not significant, around $1700 \mathrm{~Hz}$ is slightly over predicted. Such a discrepancy may be attributed to the ignored rotational FRFs at the tool-holder interface.

\subsection{0-mm diameter end mill}

In the second case, a $10-\mathrm{mm}$ diameter four-fluted end mill is considered for prediction of the tool tip FRFs. The product code of the tool is Sandvik 1P360-1000-XA 1620. The tool has $80 \mathrm{~mm}$ overhang length, where the length of the section inside the tool holder is $20 \mathrm{~mm}$. The simulations are plotted in Fig. 17 together with the experimental measurements, where a clearly good agreement is observed. The modes contributed by the robotic platform are predicted well in terms of the natural frequency but the amplitude. The first dominant mode contributed by the cutting tool is predicted and measured at 1136 and $1157 \mathrm{~Hz}$ in $X$ and $Y$ directions, respectively. The second natural frequency contributed by the cutting tool is under estimated in terms of the natural frequency, whereas amplitude wise it is in good agreement. The discrepancies observed at the higher frequency modes can attributed to the ignored rotational FRFs at the tool-holder interface.
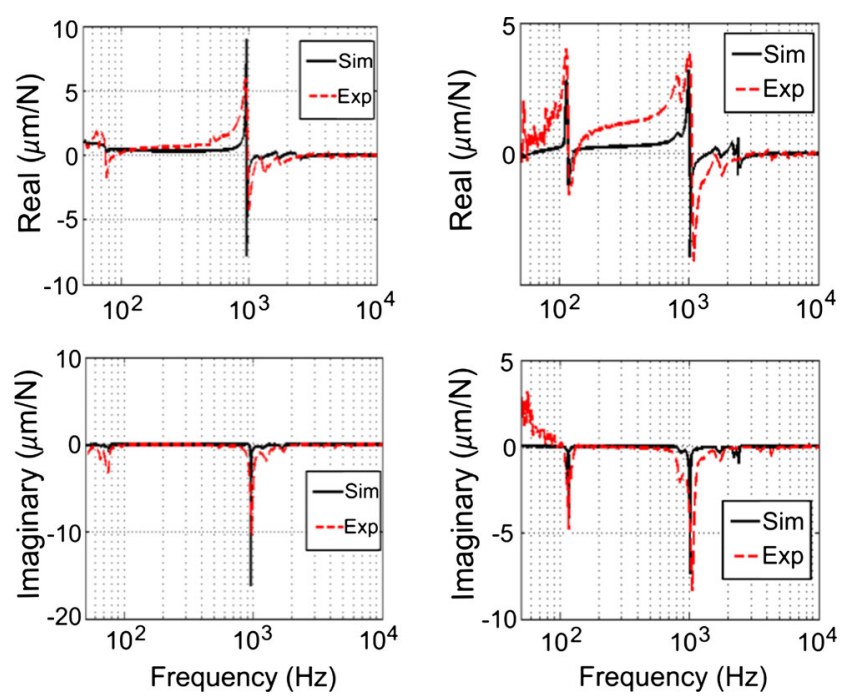

(a) Gxx

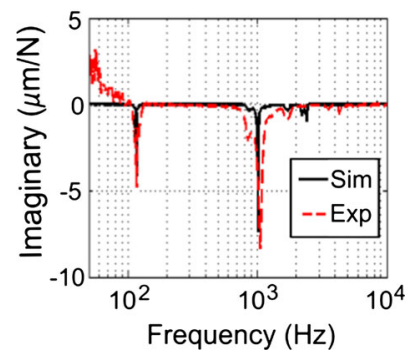

(b) Gyy

Fig. 16 Predicted and measured FRF of D18 mm end mill with $105 \mathrm{~mm}$ overhang

\subsection{2-mm diameter taper ball end mill}

The tool tip dynamics of a tapered ball end mill is simulated in the third case. The cutting tool has $12 \mathrm{~mm}$ of shank diameter and $7.989 \mathrm{~mm}$ cutting diameter at the tip. The one-sided (half) taper angle is 3 degrees, and the flute length is $40 \mathrm{~mm}$. The product code of the cutting tool is Sandvik R216.5408040RAL40G. In this case, the cross-sectional geometry of the cutting tool continuously varies along the tool axis, which demonstrates the benefit of the proposed method even better as the varying cross-sectional properties are considered in simulation of the tool's free-free response. The tool geometry is divided into nine segments. The first segment is $32 \mathrm{~mm}$ long shank, is followed by $13 \mathrm{~mm}$ long second segment, and the remaining seven segments are $5 \mathrm{~mm}$ long. Development of the tool freefree FRF, as the segments are coupled, is given in Fig. 18a.

The predictions are compared with the simulations in Fig. 18 for $X$ and $Y$ directions. It is observed that the natural frequency of both low and high frequency modes are predicted accurately, whereas there is quite a bit of discrepancy in terms of the amplitude. The first and second modes contributed by the tool body are observed around 1500 and $1700 \mathrm{~Hz}$, respectively. The tool tip dynamics seem to be asymmetrical due to the asymmetrical low frequency dynamics contributed by the robotic machining setup.

\section{Conclusions}

In this paper, a new approach is presented for prediction of tool tip FRF based on RCSA. The geometrical properties of the cutting tool are calculated exactly through a novel, analytical and quick STL slicing algorithm, where the 3D model of the tool can be either provided by the tool manufacturer or
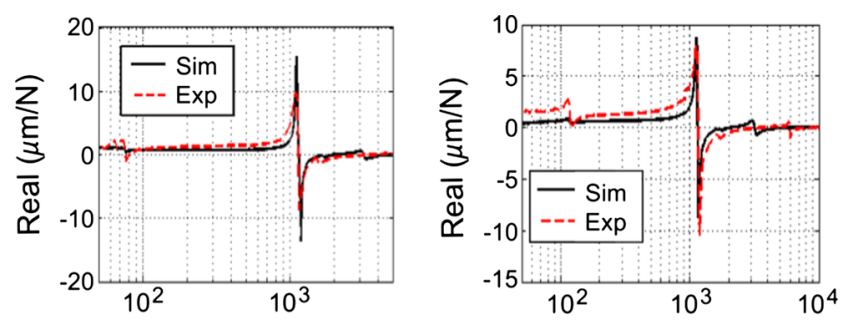

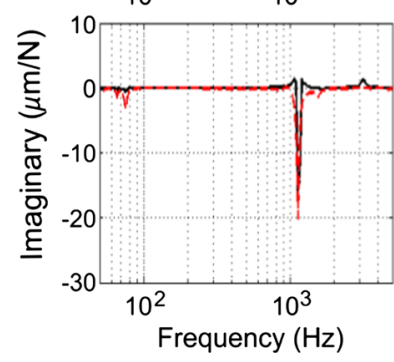

(a) Gxx

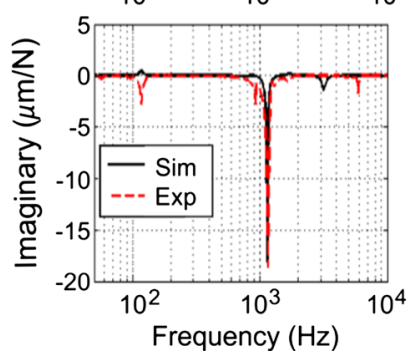

(b) Gyy
Fig. 17 D10 mm tool with $80 \mathrm{~mm}$ overhang 
Fig. 18 D12 mm taper ball end mill with $80 \mathrm{~mm}$ overhang

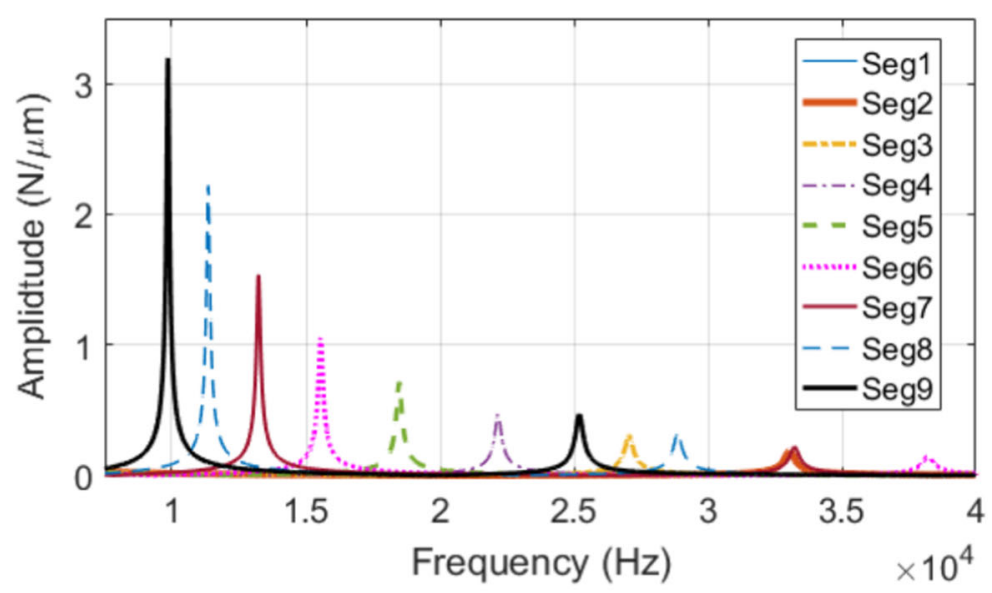

(a) Evolution of the tool free-free FRF with the coupled segments.
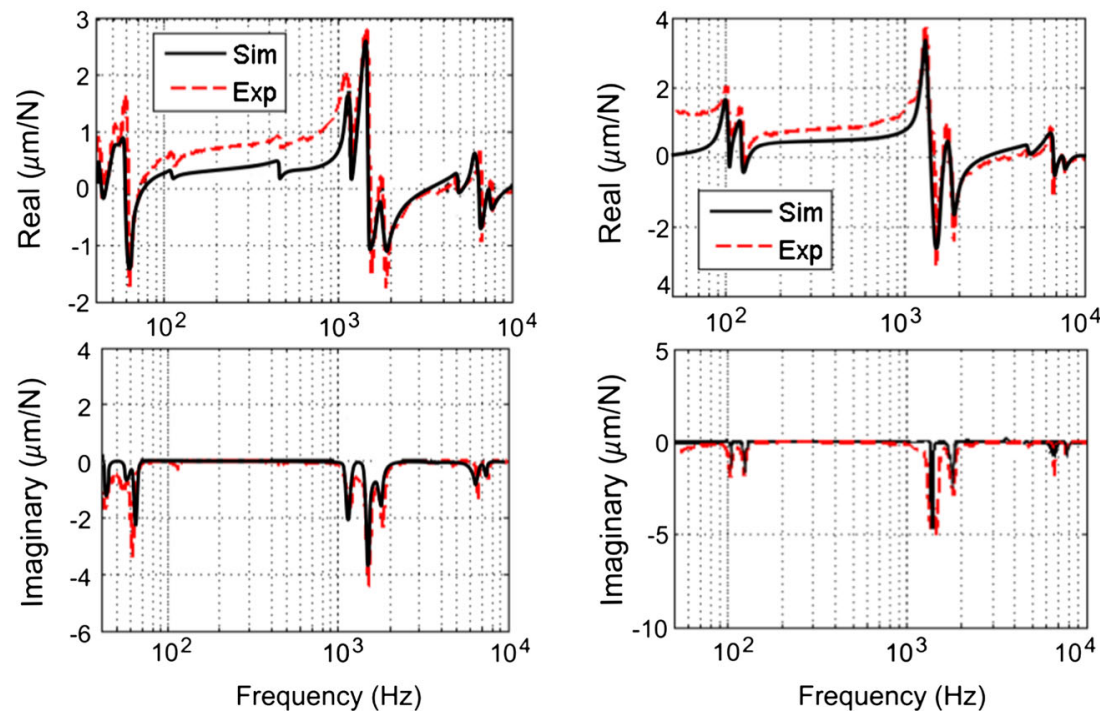

(b) Gxx

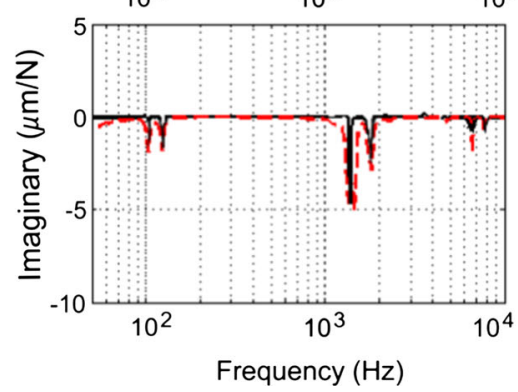

(c) Gyy obtained from a 3D measurement. The tool body is modeled as multi-segmented Timoshenko beam, which is solved through a novel iterative approach, proposed in this paper, to obtain the free-free FRF. The accuracy improvement obtained, by using the proposed STL slicing approach, in calculation of the cross-sectional properties is demonstrated on several types of milling tools. It is shown that the proposed STL slicing algorithm improves calculation accuracy especially for the tools having variable cross-sectional properties. In the cases where the tool's cross-sectional geometry continuously varies, i.e., tapered ball end mills, accurate prediction of tool tip dynamics is almost impossible using the geometrical approximation approaches proposed in the literature. In this paper, it is shown that the varying cross-sectional properties can be accommodated by slicing the STL model of the milling tool body. The prediction of free-free tool dynamics is compared to FEM simulations to demonstrate the time efficiency of the proposed approach. In regular tool cases, a computational efficiency of at least four times were observed, where for the tools with variable cross-sectional geometry it may be up to more than 20 times more efficient as FEM approaches compromise the accuracy and the computational time in a quadratic manner. The predictions are experimentally verified, where reasonable agreement is observed between simulations and impact hammer measurements both in $X$ and $Y$ directions. In some cases, small discrepancies observed which may be due to the ignored rotational FRFs. However, considering that the aim of this paper is not proposing a new receptance coupling approach, the agreement can be accepted as accurate enough to demonstrate that STL model of the tool works well for FRF prediction.

The proposed approach enables a seamless and analytical integration between the exact geometry of the cutting tool, Timoshenko beam solution and the RCSA. Thus, tool optimization studies, where recursive calculation of the tool free-free FRF may be required becomes much more practical and 
feasible in terms of calculation time. Without such an approach, use of the exact tool geometry is bounded to the FEM approaches, which requires model preparation, mesh generation, and FEM solution at every iteration.

Funding The authors gratefully acknowledge the support of EPSRC, The University of Manchester, and The University of Sheffield under NNUMAN Programme with grant number EP/J021172/1. The support of Sandvik Coromant for providing the $3 \mathrm{D}$ tool models is also acknowledged.

\section{References}

1. Altintas Y, Budak E (1995) Analytical prediction of stability lobes in milling. Ann CIRP 44:357-362

2. Insperger T, Stepan G (2002) Semi-discretization method for delayed systems. Int J Numer Methods Eng 55(5):503-518

3. Schmitz TL, Donaldson R (2000) Predicting high-speed machining dynamics by substructure analysis. Ann CIRP 49(1):303-308

4. Aristizabal-Ochoa JD (2004) Timoshenko beam column with generalized end conditions and non-classical modes of vibration of shear beams. J Eng Mech 130:1151-1159

5. Schmitz TL, Davies M, Medicus K, Snyder J (2001) Improving high speed machining material removal rates by rapid dynamic analysis. Annals of the CIRP 50(1):263-268

6. Park SS, Altintas Y, Movahhedy M (2003) Receptance coupling for end mills. Int J Mach Tools Manuf 43(9):889-896

7. Schmitz TL, Powell K, Won D, Duncan GS, Sawyer WG, Ziegert JC (2007) Shrink fit tool holder connection stiffness/damping modelling for frequency response prediction in milling. Int $\mathrm{J}$ Mach Tools Manuf 47:1368-1380

8. Namazi M, Altintas Y, Abe T, Rajapakse N (2007) Modeling and identification of tool holder-spindle interface dynamics. Int J Mach Tools Manuf 47(9):1333-1341

9. Yang Y, Wan M, Ma YC, Zhang WH (2016) An improved method for tool point dynamics analysis using a bi-distributed joint interface model. Int J Mech Sci 105:239-252
10. Özşahin O, Ertürk A, Özgüven HN, Budak E (2009) A closed-form approach for identification of dynamical contact parameters in spindle-holder-tool assemblies. Int J Mach Tools Manuf 49:25-35

11. Ertürk A, Özgüven HN, Budak E (2006) Analytical modelling of spindle-tool dynamics on machine tools using Timoshenko beam model and receptance coupling for the prediction of tool point FRF. Int J Mach Tools Manuf 46:1901-1912

12. Ertürk A, Özgüven HN, Budak E (2007) Effect analysis of bearing and interface dynamics on tool point FRF for chatter stability in machine tools by using a new analytical model for spindle-tool assemblies. Int J Mach Tools Manuf 47:23-32

13. Arbertelli P, Goletti M, Monno M (2013) A new receptance coupling substructure analysis methodology to improve chatter free cutting conditions prediction. Int J Mach Tools Manuf 72:16-24

14. Kivanc EB, Budak E (2004) Structural modeling of end mills for form error and stability analysis. Int J Mach Tools Manuf 44:1151-1161

15. Mancisidor I, Urkiola A, Barcena R, Munoa J, Dombovari Z, Zatarain M (2014) Receptance coupling for tool point dynamic prediction by fixed boundaries approach. Int J Mach Tools Manuf 78:18-29

16. Özşahin O, Altintas Y (2015) Prediction of frequency response function (FRF) of asymmetric tools from the analytical coupling of spindle and beam models of holder and tool. Int J Mach Tools Manuf 92:31-40

17. Yang Y, Zhang WH, Ma YC, Wan M (2015) Generalized method for the analysis of bending, torsional and axial receptances of toolholder-spindle assembly. Int J Mach Tools Manuf 99:48-67

18. Altintas, Y., Kersting, P., Biermann, D., Budak, E., Denkena, B., Lazoglu, I (2014) Virtual process systems for part machining operations. CIRP Ann - Manuf Technol 585-605

19. Lefebvre PP, Lauwers B (2004) STL model segmentation for multiaxis machining operations planning. Computer-Aided Design and Applications 1:277-284

20. Timoshenko SP (1922) On the transverse vibrations of bars of uniform cross-section. Philos Mag 43:125-131

21. Blevins R (1979) Formulas for natural frequency and mode shape. Van Nostrand Reinhold Co., NY 